\title{
Modal Control for Active Vibration Damping of Cable-Driven Parallel Robots
}

\author{
Loïc Cuvillon* \\ ICube laboratory \\ University of Strasbourg \\ Illkirch, 67412, France \\ Email: I.cuvillon@unistra.fr
}

\author{
Xavier Weber \\ ICube laboratory \\ University of Strasbourg \\ Illkirch, 67412, France \\ Email: mail.xavier.weber@gmail.com
}

\author{
Jacques Gangloff \\ ICube laboratory \\ University of Strasbourg \\ Illkirch, 67412, France \\ Email: jacques.gangloff@unistra.fr
}

Cable-driven parallel robots are well suited for applications that require a very large workspace. Thanks to their lightweight moving parts, they can achieve high dynamics while remaining pretty safe for nearby human workers. Furthermore, their size only depends on the length of the cables, thus their scale is almost totally decoupled from their cost. However, due to the cables, the stiffness is very low with respect to rigid link robots, inducing slowly damped oscillations of the end effector. Previous works have shown those vibrations can be effectively damped by the winch actuators thanks to active vibration damping techniques. In this paper, a gain-scheduling approach is proposed based on a linearized model of the robot dynamics. This model is projected in modal space yielding 6 decoupled transfer functions for 6 degrees of freedom of a cable-driven parallel robot using thin cables. The stability of the proposed control law is analyzed for a static and a moving end effector. The proposed control algorithm is validated experimentally on an 8-cables suspended robot prototype.

\section{Introduction}

Cable-Driven Parallel Robots (CDPR) are parallel robots using flexible cables instead of rigid sets of articulated links. This specificity allows for lightweight robots with large payloads and very large workspace. A robot with a very large workspace opens a lot of new opportunities, e.g. moving a camera over a stadium [1], moving a stretcher for rescue operations [2] or moving dexterously a heavy payload in a large warehouse [3], but several problems arise when long cables are used.

With thin cables, the CDPR stiffness is extremely low compared to a rigid link robot. With thick cables, sagging

${ }^{*}$ Corresponding author. occurs. The sagging is a deflection of the cable under its own weight, thus altering the geometrical model and reducing stiffness [4]. Low stiffness is responsible for low frequency natural vibrations of the CDPR end effector. Low frequency vibrations are especially annoying because of the long settling time of the end effector. Several approaches have been studied to tackle CDPR vibrations issues. One of them consists simply in increasing the stiffness by increasing cable tensions in order to reduce the settling time. This approach is proposed in [5] but it is limited by the admissible tension in the cables. Furthermore, a lot of power is lost for maintaining this tension. It is also unsuitable for underconstrained or suspended CDPR where some antagonist cables are lacking. The other approach is active damping of these vibrations with either embedded actuators (reactionbased mass inertia [6,7] or thrusters [8]) or with cable actuation and exteroceptive feedback [9-16].

Inspired by earlier studies on robot manipulator with flexible joints [17], Cavarly [10] and Khosravi [11] use a torque control signal of the winch actuators to ensure a stable trajectory of the CDPR. In [10], a passivity-based control is proposed based on a dynamic model that includes the end effector, the inertia of the winches and a lumped-mass model of the cables. A proportional feedback on the stretching velocity of the massless cables combined respectively with a computed torque control or a robust PID control is studied respectively in [11] and [12]. Simulations show the effectiveness of the method to track a known trajectory, while damping vibrations. Assuming high cable stiffness, i.e. that cable vibrations are fast, a proof of stability is derived using the singular perturbation theory [18]. More recently, a control law combining a PID feedback and a feed-forward term was proposed in [16]. The feed-forward aims at rejecting the end effector vibrations by inverting the elasto-dynamic model. 
Instead of using torque control of the winches actuators, Meunier [14] and Beguey [13] propose a cascade controller based on an inner loop controlling the winch angular positions. By controlling the unstretched cable length through the winding of the cable around the pulley, the elongation and thereby tension of the elastic cable can be effectively regulated. In [13], it is established how the inner position loop can efficiently cope with nonlinearites such as dry friction, usually associated with actuators with high reduction ratio gearboxes. To track a desired trajectory and reject the disturbances, an outer loop regulates the end effector Cartesian coordinates with a computed torque control using the inversion of the CDPR dynamic model and a PD controller. Pose and velocity feedback of the end effector are respectively measured with a high-speed camera and an Inertial Measurement Unit (IMU). Stability of the cascade control scheme is proved using the singular perturbation theory where the assumption of a high cable stiffness in [11] is replaced by the assumption of a high gain of the inner position loop controller. In [14], the outer loop is composed of a PID controller that rejects the joint-space position errors in order to achieve the desired end effector pose. Using an inversedynamics controller, the output of the PID controller is then converted into tension references for the inner position loop of the winches.

Lastly, Jamshidifar [15] et al. focuses on vibration rejection of a planar robot. A decoupled model of the vibration dynamics is derived from the first order linearization of the CDPR dynamics. The decoupled model is time varying, since its stiffness matrix depends on the current CDPR pose and cable tension. Thus, a linear parameter variant (LPV) controller is designed to cope with vibrations over the whole workspace. The output of the LPV controller is a tension variation that is added to the nominal tension computed using the full inverse dynamics model of the CDPR in order to track a desired trajectory. The control law is tested on a planar robot with active vibration damping along one Degree of Freedom (DoF).

In this paper, active vibration damping is considered, based on a linearized model of a CDPR along its end effector trajectory. The dynamics of the CDPR are projected in the end effector vibrations modal space. A decoupled modal controller is designed in modal space, whereas it is designed in Cartesian space in other works. The projection in the modal space converts complex coupled end effector dynamics into fully decoupled dynamics, called modes. Applying this technique to CDPRs is the main contribution of this work. It allows mainly for an easier tuning strategy: since all modes are decoupled, simple SISO controllers can be used for each mode.

The design of an active damping control algorithm usually requires a good knowledge of the CDPR vibrations characteristics. Natural frequencies and vibration modes of a CDPR are calculated from its stiffness model. Several methods have been proposed to estimate the stiffness of a CDPR as in [19-21]. A simple model proposed in [20] only relies on the knowledge of cable stiffness and CDPR geometry.
But, in some specific configurations, this model may be inaccurate and antagonistic stiffness should be considered [19].

Generic developments on modal control are presented in [22]. Modal control has been widely used for vibration damping of flexible mechanical structures. Some control algorithms like Direct Velocity, Positive Position, Integral Force Feedback and more advanced optimal control algorithms are reviewed in chapters 7 and 11 of [23]. Position and velocity feedback gains can be chosen to optimize the modal control energy [24]. Linear Quadratic Regulator (LQR) control algorithm applied to modal control is named Independent Modal Space Control (IMSC). However, so far, modal control seems to have raised relatively few interests in robotics. In [25], IMSC has been applied to a parallel robot with one flexible segment. In [26], the dynamic model of a flexible parallel robot is linearized along a path and modal analysis is used for input shaping.

Preliminary results in [27] show experimentally that a modal controller yields an effective CDPR vibration damping around a linearization point. To reject the effects of actuators nonlinearities, the winch motors are position-controlled as in $[13,14]$ and the higher level modal vibration damping loop sends set points to these inner position loops. However, the efficiency of the active damping decreases when the robot moves away from the state where the controller has been tuned. Thus, a gain-scheduling approach is considered here to generalize the active vibration damping method proposed in [27] for the whole CDPR workspace. Furthermore, a thorough analysis underlines that the stability of the proposed gain-scheduling control law is guaranteed for a moving end effector if the trajectory velocity is slow enough. Finally, the approach is validated experimentally on a new 6 DoF, 8-cables suspended robot, that shares the same cable geometry as the CoGiRo robot described in [3].

This paper is divided into 4 main sections. Section 2 describes the system, explains the assumptions that are made and defines the notations. Section 3 derives the equations of the dynamics and the modal control law. Section 4 presents a stability analysis of the proposed gain scheduling control law. Section 5 presents the experimental setup and discusses the experimental results.

\section{Context}

The approach proposed in this paper is suited for CDPRs whose cable sagging can be neglected. Sagging is the bending of a cable due to its own weight. It increases with cable linear mass and length and decreases with cable tension. There are several models for this phenomenon. A complex model (elastic catenary) is needed when the sagging is large, and a simplified (parabolic) is sufficient when it is smaller [4]. It has been shown experimentally in [21] that sagging has a significant impact on the CDPR natural frequencies and, in some configurations, a simplified model may yield very inaccurate results. Nevertheless, when sagging is negligible, the cable can be effectively modeled by a linear spring.

This model may be inaccurate for some types of cables 


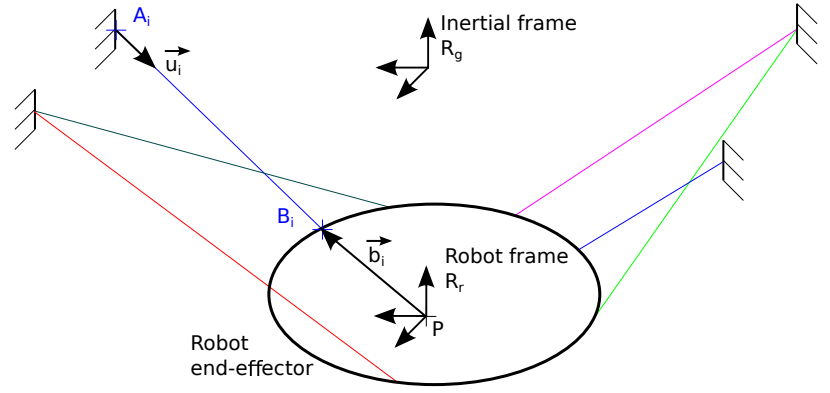

Fig. 1. CDPR notations

used to build CDPRs. Studies on cable characteristics show that cables usually have a non-linear relationship between their tension and elongation [28]. In what follows, it is assumed that this relationship can be linearized to provide a quite accurate linear spring model within nominal tension boundaries [4]. Thus, a cable is modeled as a linear spring with a specific stiffness $k_{s}=e s(\mathrm{~N})$ related to its Young modulus $e\left(\mathrm{Nm}^{-2}\right)$ and its cross-section $s\left(\mathrm{~m}^{2}\right)$. Then, the axial stiffness $k$ of the cable is given by Eq. (1).

$$
k=\frac{k_{s}}{l_{2}}=\frac{e s}{l_{2}}
$$

In Eq. (1), $l_{2}$ represents the free length of the cable, i.e. its length when its tension equal to zero. Winding cable around its pulley reduces $l_{2}$, and therefore increases the cable axial stiffness. Vibrations occurring on CDPRs are mainly due to the axial stiffness of the cables.

\section{Modeling and Control}

With modal analysis theory, the dynamics of a multiple DoF system can be split in decoupled modes, whose characteristics (frequency, direction) depend on the equilibrium point. The dynamic model of a CDPR linearized in the vicinity of its equilibrium point and projected in modal space is derived in this section. It is then used to tune a vibration rejection modal controller.

\subsection{CDPR Modeling}

Let $\mathbf{x}=\left[\begin{array}{ll}\mathbf{p}^{T} & \boldsymbol{\phi}^{T}\end{array}\right]^{T}$ be the pose vector of the CDPR end effector with respect to the base frame $R_{g}$ (Fig. 1). Vector $\phi$ contains angular coordinates (e.g. Euler angles) of the rotation $\mathbf{R}_{g r}(\boldsymbol{\phi})$ from the inertial frame $R_{g}$ to the end effector frame $R_{r}$.

Assuming massless cables, the dynamics of a CDPR end effector relatively to the inertial frame $R_{g}$ can be described by Newton-Euler equations. This model has been used and derived in many works $[13,29]$ and yields the following equation:

$$
\mathbf{M}(\mathbf{x}) \ddot{\mathbf{x}}+\mathbf{C}(\dot{\mathbf{x}}, \mathbf{x}) \dot{\mathbf{x}}+\mathbf{g}(\mathbf{x})=-\tilde{\mathbf{J}}^{T}(\mathbf{x}) \tau+\boldsymbol{\psi}
$$

where $\mathbf{M}(\mathbf{x})$ is the mass and inertia matrix, $\mathbf{C}(\dot{\mathbf{x}}, \mathbf{x})$ is the Coriolis and centrifugal matrix and $\mathbf{g}(\mathbf{x})$ is the vector of the gravitational forces. The vector $\tau$ denotes the cable tensions, while the vector $\boldsymbol{\psi}$ is an external wrench disturbance (wind, impact, ...) applied to the end effector.

The Jacobian matrice $\tilde{\mathbf{J}}$ maps the time derivative of the pose $\dot{\mathbf{x}}$ to joint velocities $\dot{\mathbf{I}}_{1}=\left[\cdots \dot{l}_{1_{i}} \cdots\right]^{T}$, i.e. the time derivative of distance $A_{i} B_{i}$ between cables ends points:

$$
\tilde{\mathbf{J}}=\left[\frac{\partial \mathbf{l}_{1}}{\partial \mathbf{x}}\right]=\mathbf{J} \mathbf{S}^{\prime}=\mathbf{J}\left[\begin{array}{cc}
\mathbb{I}_{3 \times 3} & \mathbf{0} \\
\mathbf{0} & \mathbf{S}(\boldsymbol{\phi})
\end{array}\right]
$$

where $\mathbf{S}(\boldsymbol{\phi})$ links the time derivative of $\boldsymbol{\phi}$ to the rotational velocity vector $\boldsymbol{\omega}, \boldsymbol{\omega}=\mathbf{S}(\boldsymbol{\phi}) \dot{\boldsymbol{\phi}}$, and $\mathbf{J}$ is the inverse Jacobian of the robot linking the velocity screw of the end effector to the joint velocities $\dot{\mathbf{i}}_{1}$. The inverse Jacobian transpose of a CDPR is given by:

$$
\mathbf{J}^{T}(\mathbf{x})=\left[\begin{array}{lrr}
\cdots & { }^{g} \mathbf{u}_{i} & \cdots \\
\cdots & { }^{g} \mathbf{b}_{i} \times{ }^{g} \mathbf{u}_{i} & \cdots
\end{array}\right]
$$

with $\mathbf{u}_{i}$ the unit vector giving the direction of the $i^{\text {th }}$ cable from its end point on the base frame to its end point on the end effector and $\mathbf{b}_{i}=\mathbf{P} \mathbf{B}_{i}$ the vector from the end effector center of gravity $P$ to the end point $B_{i}$ of cable $i$ on the end effector, expressed in the base frame (see Fig. 1).

With the assumption of elastic massless cables, forces applied by the cables to the end effector can be computed using the distances $\mathbf{I}_{1}$ between cable endpoints and the cable free lengths $\mathbf{I}_{2}$ (the length of the cable with no tension). The free length can be calculated using the winch angular positions $\boldsymbol{\theta}: \mathbf{l}_{2}=r \boldsymbol{\theta}$ where $r$ is a scalar representing the pulley radius. Note that $\mathbf{l}_{1}$ depends only on $\mathbf{x}$ since it is obtained by the inverse kinematics of the robot. The diagonal matrix of cables axial stiffness $\mathbf{K}=\operatorname{diag}\left(k_{i}\right)$ links cable tensions $\boldsymbol{\tau}$ to cable lengths:

$$
\boldsymbol{\tau}=\mathbf{K}\left(\mathbf{l}_{2}\right)\left[\mathbf{l}_{1}(\mathbf{x})-\mathbf{l}_{2}\right]
$$

with $k_{i}$ the stiffness of the $i^{\text {th }}$ cable depending on its free length $l_{2_{i}}$ (Eq. (1)). It is assumed that cable tension remains positive thanks to a dedicated tension management algorithm $[30,31]$.

Finally, from Eq. (2), the model of a CDPR dynamics with massless elastic cables becomes:

$$
\mathbf{M}(\mathbf{x}) \ddot{\mathbf{x}}+\mathbf{C}(\dot{\mathbf{x}}, \mathbf{x}) \dot{\mathbf{x}}+\mathbf{g}(\mathbf{x})=\tilde{\mathbf{J}}^{T} \mathbf{K}\left(\mathbf{l}_{2}\right)\left[\mathbf{l}_{2}-\mathbf{l}_{1}(\mathbf{x})\right]+\boldsymbol{\psi}
$$

\subsection{Modal Analysis}

Modal analysis is a method for decomposing the oscillatory response of a mechanical structure around an equilibrium point into elementary decoupled sinusoid components called "modes".

A linearized model of the CDPR dynamics (Eq. (6)) in the vicinity of a feasible or equilibrium trajectory 
$\left(\ddot{\ddot{\mathbf{x}}}(t), \dot{\overline{\mathbf{x}}}(t), \overline{\mathbf{x}}(t), \overline{\mathbf{l}}_{2}(t), \bar{\psi}(t)\right)$ is obtained by a first-order Taylor expansion (see appendix A for a detailed derivation) :

$$
\begin{aligned}
& \mathbf{M}(\overline{\mathbf{x}}) \Delta \ddot{\mathbf{x}}+\left[\mathbf{C}(\dot{\overline{\mathbf{x}}}, \overline{\mathbf{x}})+\left.\frac{\partial \mathbf{C}(\dot{\mathbf{x}}, \mathbf{x})}{\partial \dot{\mathbf{x}}}\right|_{\dot{\mathbf{x}}, \overline{\mathbf{x}}} \dot{\overline{\mathbf{x}}}\right] \Delta \dot{\mathbf{x}} \\
- & {\left[\tilde{\mathbf{J}}^{T}(\overline{\mathbf{x}}) \operatorname{diag}^{-1}\left(\overline{\mathbf{l}_{2}}\right) \operatorname{diag}(\overline{\boldsymbol{\tau}})+\tilde{\mathbf{J}}^{T}(\overline{\mathbf{x}}) \mathbf{K}\left(\overline{\mathbf{l}}_{2}\right)\right] \Delta \mathbf{l}_{2} } \\
+ & {\left[\left.\frac{\partial \mathbf{M}(\mathbf{x})}{\partial \mathbf{x}}\right|_{\overline{\mathbf{x}}} \ddot{\mathbf{x}}+\left.\frac{\partial \mathbf{C}(\dot{\mathbf{x}}, \mathbf{x})}{\partial \mathbf{x}}\right|_{\dot{\mathbf{x}}, \overline{\mathbf{x}}} \dot{\overline{\mathbf{x}}}+\left.\frac{\partial \tilde{\mathbf{J}}^{T}(\mathbf{x})}{\partial \mathbf{x}}\right|_{\overline{\mathbf{x}}} \bar{\tau}\right.} \\
+ & \left.\tilde{\mathbf{J}}^{T}(\overline{\mathbf{x}}) \mathbf{K}\left(\overline{\mathbf{l}}_{2}\right) \tilde{\mathbf{J}}(\overline{\mathbf{x}})\right] \Delta \mathbf{x}-\Delta \psi
\end{aligned}
$$

with $\Delta \mathbf{x}=\mathbf{x}-\overline{\mathbf{x}}$, the difference between the current pose and the equilibrium one, and $\overline{\boldsymbol{\tau}}=\mathbf{K}\left(\overline{\mathbf{l}}_{2}\right)\left[\mathbf{l}_{1}(\overline{\mathbf{x}})-\overline{\mathbf{l}}_{2}\right]$ (Eq. (5)).

Considering a static equilibrium $(\ddot{\overline{\mathbf{x}}}=\mathbf{0}, \dot{\overline{\mathbf{x}}}=\mathbf{0})$ of the CDPR end effector, the linearized model of the CDPR dynamics is reduced to :

$$
\begin{array}{r}
\mathbf{M}(\overline{\mathbf{x}}) \Delta \ddot{\mathbf{x}}+\mathbf{K}_{\mathbf{x}}\left(\overline{\mathbf{x}}, \overline{\mathbf{l}}_{2}\right) \Delta \mathbf{x} \\
=\mathbf{T}\left(\overline{\mathbf{x}}, \overline{\mathbf{l}}_{2}\right) \Delta \mathbf{l}_{2}+\Delta \boldsymbol{\Psi}
\end{array}
$$

with

$$
\begin{array}{r}
\mathbf{K}_{\mathbf{x}}\left(\overline{\mathbf{x}}, \overline{\mathbf{l}}_{2}\right)=\left.\frac{\partial \tilde{\mathbf{J}}^{T}(\mathbf{x})}{\partial \mathbf{x}}\right|_{\overline{\mathbf{x}}} \bar{\tau}+\tilde{\mathbf{J}}^{T}(\overline{\mathbf{x}}) \mathbf{K}\left(\overline{\mathbf{l}}_{2}\right) \tilde{\mathbf{J}}(\overline{\mathbf{x}}) \\
\mathbf{T}\left(\overline{\mathbf{x}}, \overline{\mathbf{l}}_{2}\right)=\tilde{\mathbf{J}}^{T}(\overline{\mathbf{x}}) \mathbf{K}\left(\overline{\mathbf{l}}_{2}\right) \operatorname{diag}\left(\mathbf{l}_{1}(\overline{\mathbf{x}})\right) \operatorname{diag}^{-1}\left(\overline{\mathbf{l}}_{2}\right)
\end{array}
$$

The matrix $\mathbf{K}_{\mathbf{x}}$ is the Cartesian stiffness of the robot, i.e. the relationship between elementary Cartesian displacements of the end effector and corresponding variations of the wrench applied to the end effector by the cables. It is symmetric if no external moment $(\overline{\boldsymbol{\psi}}=0)$ is applied to the end effector [32]. Furthermore, $\mathbf{K}_{\mathbf{x}}$ is positive definite except in a few singular situations detailed in [19].

Based on the linear model (Eq. (8)), a classic modal analysis is applied to convert the coupled system into six single DOF linear systems. Let $\boldsymbol{\eta}$ be the six dimensional vector of modal coordinates. Those coordinates are related to the pose variation $\Delta \mathbf{x}$ by the $6 \times 6$ modal transformation matrix $\mathbf{E}$ :

$$
\Delta \mathbf{x}=\mathbf{E} \boldsymbol{\eta}
$$

The modal matrix $\mathbf{E}$ has to verify the following conditions:

$$
\left\{\begin{array}{l}
\mathbf{E}^{T} \mathbf{M E}=\mathbb{I}_{6 \times 6} \\
\mathbf{E}^{T} \mathbf{K}_{\mathbf{x}} \mathbf{E}=\boldsymbol{\Lambda}
\end{array}\right.
$$

where $\mathbb{I}_{6 \times 6}$ is the $6 \times 6$ identity matrix and $\Lambda$ is the $6 \times 6$ diagonal matrix of the system eigenvalues.
The matrix $\mathbf{E}$ is obtained by decomposition in generalized eigenvalues $\boldsymbol{\Lambda}$ of the system verifying the following equation:

$$
\mathbf{K}_{\mathbf{x}} \mathbf{E}=\mathbf{M E} \boldsymbol{\Lambda}
$$

As $\mathbf{K}_{\mathbf{x}}$ and $\mathbf{M}$ are symmetric, $\mathbf{E}^{T} \mathbf{K}_{\mathbf{x}} \mathbf{E}$ and $\mathbf{E}^{T} \mathbf{M E}$ are both diagonal matrices [23]. Each column eigenvector $\mathbf{e}_{i}$ of $\mathbf{E}=$ $\left[\mathbf{e}_{1}, \mathbf{e}_{2}, \ldots, \mathbf{e}_{6}\right]$ can be normalized such that $\mathbf{e}_{i}{ }^{T} \mathbf{M} \mathbf{e}_{i}=1$ in order to verify the first condition of Eq. (12). This normalization is possible since if one $\mathbf{e}_{i}$ is solution of the generalized eigenvalues problem, i.e. $\mathbf{K}_{\mathbf{x}} \mathbf{e}_{i}=\mathbf{M e}_{i} \lambda_{i}$, then the vector $\alpha_{i} \mathbf{e}_{i}$ is also a solution with $\alpha_{i}$ an arbitrary constant.

Rewriting Eq. (8) with modal coordinates (Eq. (11)) yields:

$$
\mathbf{M E} \ddot{\eta}+\mathbf{K}_{\mathbf{x}} \mathbf{E} \boldsymbol{\eta}=\mathbf{T} \Delta \mathbf{l}_{2}+\Delta \psi
$$

Left product by $\mathbf{E}^{T}$ gives:

$$
\mathbb{I}_{6 \times 6} \ddot{\boldsymbol{\eta}}+\boldsymbol{\Lambda}\left(\overline{\mathbf{x}}, \overline{\mathbf{l}}_{2}\right) \boldsymbol{\eta}=\mathbf{E}\left(\overline{\mathbf{x}}, \overline{\mathbf{l}}_{2}\right)^{T}\left[\mathbf{T}\left(\overline{\mathbf{x}}, \overline{\mathbf{l}}_{2}\right) \Delta \mathbf{l}_{2}+\Delta \boldsymbol{\psi}\right]
$$

Left side of equation (15) is fully decoupled and so can be decomposed into 6 independent equations, one for each mode. Matrix $\mathbf{E}$ contains 6 vectors of vibration modes and diagonal matrix $\Lambda$ contains 6 squared natural frequencies corresponding to these modes. Therefore in this particular space, the response of the CDPR to a joint position variation $\Delta \mathbf{l}_{2}$ or an external wrench disturbance $\Delta \psi$ is a vector of six independent sinusoids.

\subsection{Control Architecture and Decoupled SISO Model of the System}

Let us consider an equilibrium state of the CDPR defined by $\left(\overline{\mathbf{x}}, \overline{\mathbf{l}}_{2}\right)$ : the CDPR pose $\overline{\mathbf{x}}$ and the associated free cable lengths $\overline{\mathbf{l}}_{2}$ that unequivocally define the cable tensions (Eq. (5)). The goal of the vibration damping algorithm is to regulate towards zero the end effector velocity $\dot{\mathbf{x}}$ as fast as possible. To achieve this rejection, the controller acts on the cable tensions through a variation of the unconstrained cable length $\Delta \mathbf{l}_{2}$ around $\overline{\mathbf{l}}_{2}$. The general architecture of the disturbance rejection control loop is presented in Fig. 2.

Using pulley actuators to wind or unwind unconstrained cable length, the control signal is equivalent to a pulley angular position variation $\Delta \boldsymbol{\theta}$. An inner winch position loop can efficiently cope with nonlinearites such as dry friction, usually associated with actuators with high reduction ratio gearboxes. In [13], a stability analysis using singular perturbation theory shows that, if the gain of the inner position loop is high enough, the stability of the outer CDPR pose loop is not compromised by the inner loop dynamics. Assuming a high-gain inner position loop, its high-bandwidth dynamics are negligible with respect to the low frequency CDPR oscillatory modes and thus are omitted thereafter. 


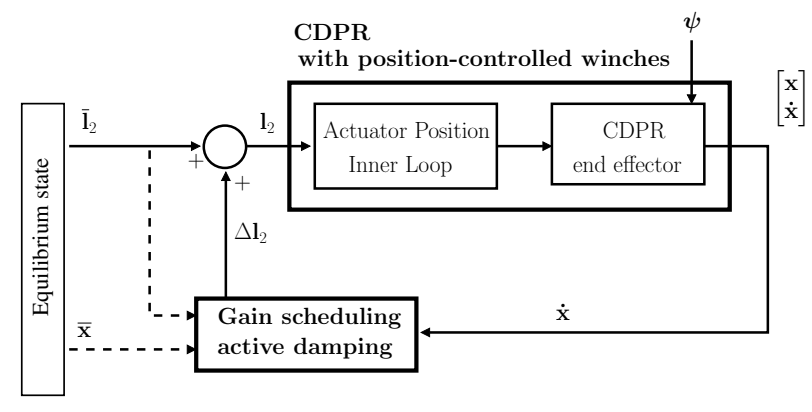

Fig. 2. Architecture of the vibration damping control loop

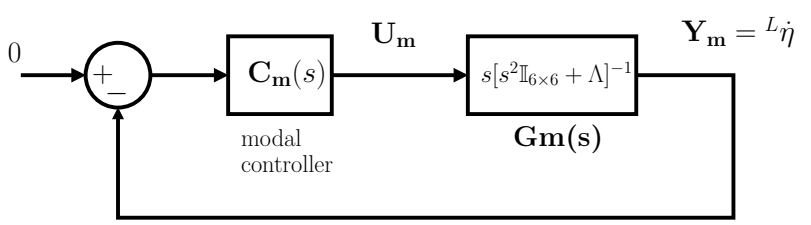

Fig. 3. Vibration damping control loop scheme in modal space

Regulating $\Delta \dot{\mathbf{x}}$ toward zero, or equally $\dot{\mathbf{x}}$ (since at equilibrium $\dot{\overline{\mathbf{x}}}=0$ ), is equivalent to regulate $\dot{\boldsymbol{\eta}}$ towards zero in modal space, since $\Delta \dot{\mathbf{x}}=\mathbf{E} \dot{\boldsymbol{\eta}}$. As the vibration problem can be decoupled in the modal space (Eq. (15)), simple SingleInput Single-Output (SISO) control strategies can be used for active vibration damping. Setting $\Delta \boldsymbol{\psi}=0$, accordingly to the superposition theorem, and applying the Laplace transform to Eq. (15) yields:

$$
\begin{aligned}
& s^{2} \mathbb{I}_{6 \times 6}{ }^{L} \boldsymbol{\eta}+\boldsymbol{\Lambda}^{L} \boldsymbol{\eta}=\mathbf{E}^{T} \mathbf{T}{ }^{L} \Delta \mathbf{l}_{2} \\
& { }^{L} \dot{\boldsymbol{\eta}}=s\left[s^{2} \mathbb{I}_{6 \times 6}+\boldsymbol{\Lambda}\right]^{-1} \underbrace{\mathbf{E}^{T} \mathbf{T}{ }^{L} \Delta \mathbf{l}_{2}}_{{ }_{\mathbf{u}_{\mathbf{m}}}}
\end{aligned}
$$

where ${ }^{L}\{$.$\} stands for the Laplace transform and s$ is the Laplace variable. Finally, let $\mathbf{Y}_{\mathbf{m}}={ }^{{ }} \dot{\boldsymbol{\eta}}$ and $\mathbf{U}_{\mathbf{m}}={ }^{{ }^{L}} \mathbf{u}_{\mathbf{m}}$ be the modal control signal for the regulation defined by $\mathbf{U}_{\mathbf{m}}=\mathbf{E}^{T} \mathbf{T}{ }^{L} \Delta \mathbf{l}_{2}$. The decoupled dynamics of the CDPR in modal space (Eq. (17)) are modeled by a diagonal transfer function matrix $\mathbf{G}_{\mathbf{m}}$ :

$$
\begin{aligned}
\mathbf{Y}_{\mathbf{m}}(\mathbf{s}) & =s\left[s^{2} \mathbb{I}_{6 \times 6}+\boldsymbol{\Lambda}\right]^{-1} \mathbf{U}_{\mathbf{m}}(\mathbf{s}) \\
& \triangleq \mathbf{G}_{\mathbf{m}} \mathbf{U}_{\mathbf{m}}(\mathbf{s})
\end{aligned}
$$

The transfer function $\mathbf{G}_{\mathbf{m}}(s)=s\left[s^{2} \mathbb{I}_{6 \times 6}+\boldsymbol{\Lambda}\right]^{-1}$ is a $6 \times 6$ diagonal matrix of transfer functions with undamped second order dynamics, consistent with the fact that frictions are neglected. The regulation loop based on this model is described in Fig. 3. The controller $\mathbf{C}_{\mathbf{m}}$ (also a diagonal matrix of transfer functions) can be designed and tuned to efficiently regulate towards zero the modal velocity $\mathbf{Y}_{\mathbf{m}}$. So, the $6 \times 6$ Multiple-Input Multiple-Output (MIMO) coupled model in Cartesian space can be easily controlled using 6 independent SISO controllers in modal space.

The implementation details of the modal control loop are given in Fig. 4. Based on the definition of the modal control signal $\mathbf{u}_{\mathbf{m}}$, the output of the modal controller is back projected into the joint space using $\mathbf{T}^{+}$, the right pseudoinverse of $\mathbf{T}$, yielding the control signal $\Delta \mathbf{l}_{2}=\mathbf{T}^{+}\left(\mathbf{E}^{T}\right)^{-1} \mathbf{u}_{\mathbf{m}}$. The modal velocity, evaluated by projecting the end effector velocity $\dot{\mathbf{x}}$ in the modal space with the matrix $\mathbf{E}^{-\mathbf{1}}$, is fed back to the controller: $\dot{\boldsymbol{\eta}}=\mathbf{E}^{-1} \Delta \hat{\hat{\mathbf{x}}}=\mathbf{E}^{-1} \hat{\hat{\mathbf{x}}}$. The estimate of $\dot{\mathbf{x}}$, denoted $\hat{\hat{\mathbf{x}}}$, is measured thanks to an IMU (Inertial Measurement Unit, a 3-axis accelerometer combined with a 3axis gyrometer). Since $\mathbf{T T}^{+}=\mathbb{I}_{6 \times 6}$ by definition, the block diagram in Fig. 4 is equivalent to the modal loop in Fig. 3.

Modal analysis assumes that $\overline{\mathbf{x}}$ stays constant. So the proposed modal stabilization control law should be restricted to the rejection of external disturbances (transient external forces applied to the end effector by the environment, like wind blowing or contact) around a static pose. In the upcoming sections, it will be shown that the proposed control law remains stable even with a slowly varying $\overline{\mathbf{x}}$. Thus, this approach allows for rejection of oscillations even during a slow trajectory.

In order to track a varying pose $\overline{\mathbf{x}}$, a corresponding cable tension $\bar{\tau}$ can be computed using the CDPR inverse dynamics (Eq. (2)) and the desired trajectory parameters $(\overline{\mathbf{x}}, \dot{\overline{\mathbf{x}}}, \ddot{\overline{\mathbf{x}}})$ :

$$
\begin{aligned}
\overline{\boldsymbol{\tau}} & =\left(\tilde{\mathbf{J}}^{T}(\overline{\mathbf{x}})\right)^{+}[\mathbf{M}(\overline{\mathbf{x}}) \ddot{\overline{\mathbf{x}}}+\mathbf{C}(\dot{\overline{\mathbf{x}}}, \overline{\mathbf{x}}) \dot{\overline{\mathbf{x}}}+\mathbf{G}]+\boldsymbol{\tau}_{\text {Null }} \\
& =\boldsymbol{\tau}+\boldsymbol{\tau}_{\text {Null }}
\end{aligned}
$$

where $\left(\tilde{\mathbf{J}}^{T}\right)^{+}$stands for the Moore-Penrose pseudoinverse of the wrench matrix, $\boldsymbol{\tau}$ is the tension solution of Eq. (2) with minimal norm, and $\boldsymbol{\tau}_{\text {Null }} \in \operatorname{Null}\left(\tilde{\mathbf{J}}^{T}\right)$ such that $\tilde{\mathbf{J}}^{T} \boldsymbol{\tau}_{\text {Null }}=0$. In case of a redundant CDPR (more cables than degrees of freedom), the vector $\tau_{\text {Null }}$ can be selected in the null space of the wrench matrix to ensure positive tension of the cables $(\bar{\tau}>0)$. Various algorithms $[30,31]$ are proposed in the literature to select an adequate $\boldsymbol{\tau}_{\text {Null }}$ vector.

Since the wrench applied on the CDPR end effector results from modifications of the cable elongation, the cable tensions are converted into unconstrained cable length $\overline{\mathbf{I}}_{2}$. Based on the linear elastic cable model (Eq. (5)), $\overline{\mathbf{I}}_{2}$ is given by:

$$
\overline{\mathbf{l}}_{2}=\left[\mathbb{I}+\frac{1}{k_{s}} \operatorname{diag}(\underbrace{\boldsymbol{\tau}+\boldsymbol{\tau}_{\text {Null }}}_{\bar{\tau}})\right]^{-1} \mathbf{l}_{1}(\overline{\mathbf{x}})
$$

Note that the positive tension condition $(\bar{\tau}>0)$ implies $\overline{\mathbf{l}}_{2}<\mathbf{l}_{1}$, i.e. the cable is stretched. The complete trajectory generation for a varying $\overline{\mathbf{x}}$ is described in Fig. 5 . 
CDPR with position-controlled winches

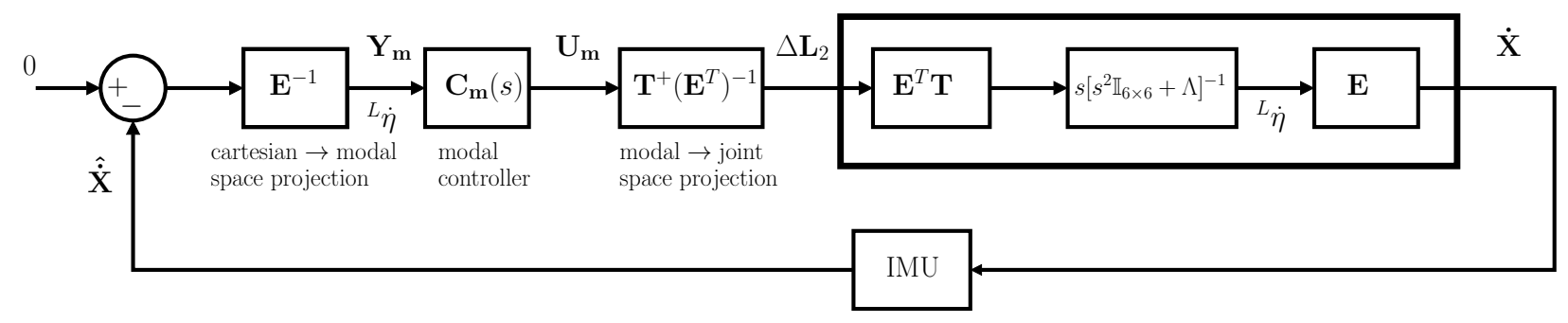

Fig. 4. Practical implementation of the modal vibration damping control on the CDPR

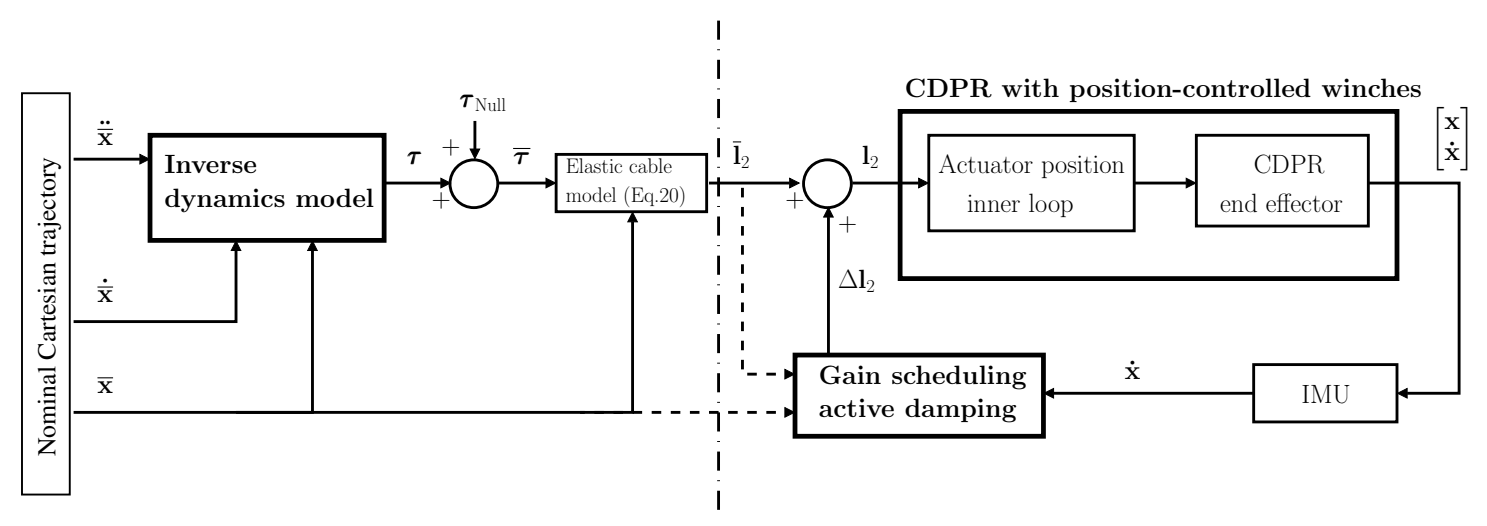

Fig. 5. Block diagram of the CDPR nominal trajectory generation (left side) combined with the vibration damping control (right side)

\subsection{Gain Scheduling Control Law}

In modal space, the open-loop transfer function $\mathbf{G}_{\mathbf{m}}$ depends only on the current $\boldsymbol{\Lambda}\left(\overline{\mathbf{x}}, \overline{\mathbf{l}}_{2}\right)$ (Eq. (18)). This transfer function is simple enough in modal space to find an algebraic pole placement law for the controller that guarantees constant disturbance rejection performances over the whole workspace using gain scheduling. So, a basic pole placement approach can be used to tune 6 SISO controllers $C_{m_{i}}$ in $\mathbf{C}_{\mathbf{m}}=\operatorname{diag}\left(C_{m_{1}}, \ldots, C_{m_{6}}\right)$ according to the 6 SISO open-loop transfer functions $G_{m_{i}}$ in $\mathbf{G}_{\mathbf{m}}=\operatorname{diag}\left(G_{m_{1}}, \ldots, G_{m_{6}}\right)$.

Model $G_{m_{i}}$ has an invariant structure but has varying parameters since it has two pure imaginary conjugate poles that depends on the frequency of the corresponding mode in $\boldsymbol{\Lambda}$. So, for given tuning specifications, the structure of the controller should also be constant.

A classical strategy when doing pole placement is to cancel stable system poles/zeros with controller zeros/poles. However, applied to the modal conjugate poles, this approach would yield a controller with a $0 \mathrm{~dB}$ gain at the system modal frequency (notch filter). It means that such a controller would be unable to reject modal oscillations due to external disturbances. Obviously, this is not the kind of behavior that is expected. Instead, for each mode, a controller is tuned for fast disturbance rejection and measurement noise resilience.

Two complex conjugate poles and one zero are added to the controller to place the four closed loop poles like in the root locus of Fig. 6. The controller gain $k_{c_{i}}$ is tuned in order to obtain a closed loop with two identical pairs of complex conjugate poles. The complex conjugate poles and the zero of the controller have the same negative real part $-a$ and

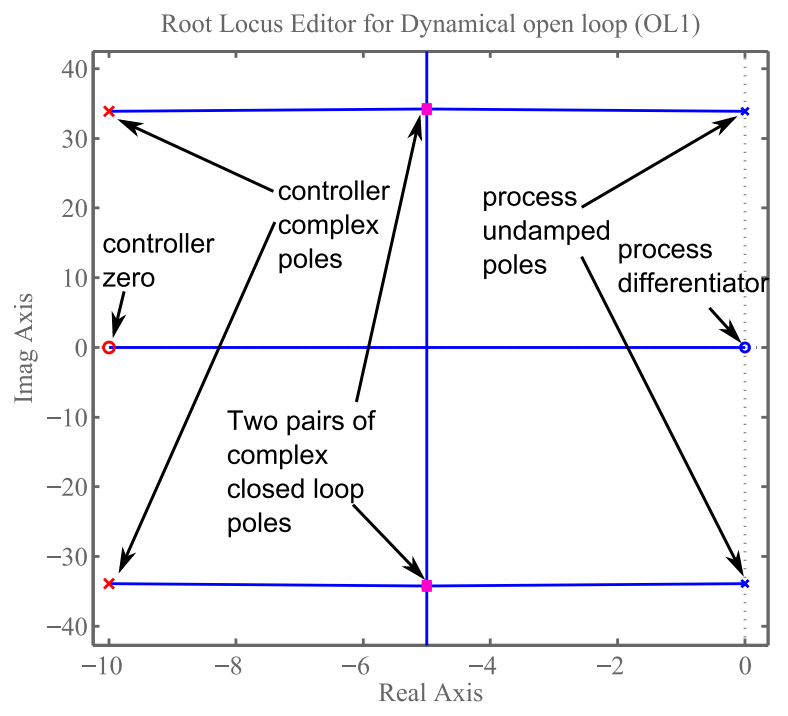

Fig. 6. Root locus design of a modal controller

the imaginary part of the controller complex poles is taken equal to the imaginary part of the process complex poles, i.e. $\sqrt{\Lambda_{i}}$ where $\Lambda_{i}$ is the $i^{t h}$ eigenvalue in $\Lambda$. With this design, measurement noise is well filtered and the controller is robust to small modeling errors. At the end, the modal controller for the $i^{\text {th }}$ modal velocity has the form:

$$
C_{m_{i}}=\frac{k_{c_{i}}(s+a)}{s^{2}+2 a s+\left(a^{2}+\Lambda_{i}\right)}
$$


with $k_{c_{i}}=2\left(\sqrt{\Lambda_{i}\left(a^{2}+\Lambda_{i}\right)}-\Lambda_{i}\right)$.

The negative real part $-a$ of the controller poles and zero is the only design parameter. Increasing $a$ yields a faster closed loop, but at the cost of a higher control signal energy. Thus, a trade-off must be found to avoid actuator saturation. With $a \in] 0,2 \Lambda_{i} \sqrt{2+\sqrt{5}}$, the two pairs of closed loop complex conjugate poles are:

$$
-\frac{a}{2} \pm j \sqrt{\sqrt{\Lambda_{i}\left(a^{2}+\Lambda_{i}\right)}-\frac{a^{2}}{4}}
$$

Since the real part $-a / 2$ of the closed loop poles is negative, the system is stable. So, with a reference equal to zero, the modal velocity $\dot{\eta}$ converges towards zero. An implementation of this approach on the CoMiRo robot is presented in section 5 .

\section{Stability Analysis}

\subsection{Near the Equilibrium Point}

A static equilibrium pose of the CDPR is fully defined by $\left(\overline{\mathbf{x}}, \overline{\mathbf{l}}_{2}\right)$. While $\overline{\mathbf{x}}$ is the pose of the end effector, $\overline{\mathbf{l}}_{2}$ is one solution of the inverse kinetostatic (IKS) problem which fully defines the cable tensions and verifies $\mathbf{G}(\overline{\mathbf{x}})$ $\tilde{\mathbf{J}}^{T} \mathbf{K}\left(\overline{\mathbf{l}}_{2}\right)\left[\overline{\mathbf{l}}_{2}-\mathbf{l}_{1}(\overline{\mathbf{x}})\right]=\mathbf{0}$ in Eq. (6). Around this equilibrium point, the CDPR dynamic model is parameterized by $\boldsymbol{\Lambda}\left(\overline{\mathbf{x}}, \overline{\mathbf{l}}_{2}\right)$ in the modal space with:

$$
\ddot{\eta}+\Lambda \eta=u_{m}
$$

and a generic state space representation of the previous decoupled modal controller $\mathbf{C}_{\mathbf{m}}$ is:

$$
\left\{\begin{array}{l}
\dot{\mathbf{x}}_{c}=\mathbf{F}_{c}(\Lambda) \mathbf{x}_{c}+\mathbf{G}_{c}(\Lambda) \dot{\boldsymbol{\eta}} \\
\mathbf{u}_{\mathbf{m}}=\mathbf{H}_{c}(\Lambda) \mathbf{x}_{c}
\end{array}\right.
$$

with $\mathbf{x}_{c}$, the controller state vector, $\mathbf{u}_{m}$ the modal control signal, $\dot{\boldsymbol{\eta}}$, the measured modal velocities, $\mathbf{F}_{c}, \mathbf{G}_{c}, \mathbf{H}_{c}$, respectively the state, input and output matrices. Since the modes are decoupled, these matrices are all block diagonal.

The closed loop system composed of Eq. (23)-(24) is stable, since the design of the controller ensures that the poles or state matrix eigenvalues of the closed loop system have all strictly negative real parts.

The stability properties and eigenvalues of the state matrix are conserved for a linear time-invariant (LTI) system through a similarity or coordinate transformation. Thus, the stability of the closed loop in the modal space yields the stability of the closed loop in the Cartesian space due to the coordinate transformation $\boldsymbol{\eta}=\mathbf{E}^{-1} \Delta \mathbf{x}$. Using $\mathbf{M}=\left(\mathbf{E}^{T}\right)^{-1} \mathbf{E}^{-1}$ and $\mathbf{K}_{\mathbf{x}}=\left(\mathbf{E}^{T}\right)^{-1} \boldsymbol{\Lambda} \mathbf{E}^{-1}$ (Eq. (12)) and the definition of $\mathbf{u}_{\mathbf{m}}$ (Eq. (17)), the coordinate transformation of the closed loop (Eq. (23)-(24)) from the modal space to the Cartesian space yields the stable system:

$$
\begin{aligned}
& \mathbf{M}(\overline{\mathbf{x}}) \Delta \ddot{\mathbf{x}}+\mathbf{K}_{\mathbf{x}}\left(\overline{\mathbf{x}}, \overline{\mathbf{l}}_{2}\right) \Delta \mathbf{x}=\mathbf{T}\left(\overline{\mathbf{x}}, \overline{\mathbf{l}}_{2}\right) \Delta \mathbf{l}_{2} \\
& \left\{\begin{array}{l}
\Delta \mathbf{l}_{2}=\mathbf{T}^{+}\left(\mathbf{E}^{T}\right)^{-1} \mathbf{H}_{c}\left(\overline{\mathbf{x}}, \overline{\mathbf{l}}_{2}\right) \mathbf{x}_{c} \\
\dot{\mathbf{x}}_{c}=\mathbf{F}_{c}\left(\overline{\mathbf{x}}, \overline{\mathbf{l}}_{2}\right) \mathbf{x}_{c}+\mathbf{G}_{c} \mathbf{E}^{-1}\left(\overline{\mathbf{x}}, \overline{\mathbf{l}}_{2}\right) \Delta \dot{\mathbf{x}}
\end{array}\right.
\end{aligned}
$$

which is the interconnection of the linearized dynamics model of the CDPR (Eq. (8)) around the constant equilibrium $\left(\overline{\mathbf{x}}, \overline{\mathbf{l}}_{2}\right)$ and a LTI controller.

Finally, as the linearized system is stable, the Lyapunov indirect method theorem [18] points out that the interconnection of the CDPR nonlinear dynamics (Eq. (6)) and the linear controller (Eq. (26)) is asymptotically stable in a neighborhood of the constant equilibrium $\left(\overline{\mathbf{x}}, \overline{\mathbf{l}}_{2}\right)$.

\subsection{Along a Trajectory}

A continuously differentiable trajectory of the CDPR end effector pose, defined and scheduled by the exogenous input signal $\overline{\mathbf{x}}(t)$, is considered. For each point on this trajectory, it is assumed that a corresponding continuous cable length $\overline{\mathbf{l}}_{2}(t)=\mathbf{I}_{2}(\overline{\mathbf{x}}(t))$, solution of the IKS model, is known or assessed with the trajectory generation scheme in Fig. 5 when the value of $\ddot{\ddot{\mathbf{x}}}$ and $\dot{\overline{\mathbf{x}}}$ reaches zero.

In this case, the plant control input that ensures tracking of the desired trajectory and vibration damping (Fig. 5) is $\mathbf{l}_{2}(t)=\overline{\mathbf{l}}_{2}(\overline{\mathbf{x}}(t))+\Delta \mathbf{l}_{2}$ with $\Delta \mathbf{l}_{2}$, the output of the vibration damping controller (Eq. (26)). Thus, the closed loop system composed of the CDPR dynamics and the gain scheduled controller is defined by the state equations:

$$
\begin{aligned}
& \mathbf{M}(\mathbf{x}) \ddot{\mathbf{x}}+\mathbf{C}(\dot{\mathbf{x}}, \mathbf{x}) \dot{\mathbf{x}}+\mathbf{G}(\mathbf{x})=\tilde{\mathbf{J}}^{T}(\mathbf{x}) \mathbf{K}\left(\mathbf{l}_{2}\right)\left[\mathbf{l}_{2}-\mathbf{l}_{1}(\mathbf{x})\right] \\
& \left\{\begin{array}{l}
\mathbf{l}_{2}=\overline{\mathbf{l}}_{2}+\mathbf{T}^{+}\left(\mathbf{E}^{T}\right)^{-1} \mathbf{H}_{c}\left(\overline{\mathbf{x}}, \overline{\mathbf{l}}_{2}\right) \mathbf{x}_{c} \\
\dot{\mathbf{x}}_{c}=\mathbf{F}_{c}\left(\overline{\mathbf{x}}, \overline{\mathbf{l}}_{2}\right) \mathbf{x}_{c}+\mathbf{G}_{c} \mathbf{E}^{-1}\left(\overline{\mathbf{x}}, \overline{\mathbf{l}}_{2}\right) \dot{\mathbf{x}}
\end{array}\right.
\end{aligned}
$$

The system has now time varying parameters, due to the dependence of the controller on the scheduling signal $\overline{\mathbf{x}}(t)$ that defines the trajectory. Some stability properties for such a gain scheduled closed loop can be assessed using theorems dealing with the stability of nonlinear system with a "slowly" varying input $[33,34]$. Let $\overline{\mathbf{x}}(t)$ be the varying input. The system of Eq. (27)-(28) can be rewritten using the standard state-space representation:

$$
\dot{\mathbf{x}}_{c l}(t)=f\left(\mathbf{x}_{c l}(t), \overline{\mathbf{x}}(t)\right)
$$

with $\mathbf{x}_{c l}=\left[\dot{\mathbf{x}}^{T} \mathbf{x}^{T} \mathbf{x}_{c}{ }^{T}\right]^{T}$, the closed loop state vector, concatenation of the CDPR and controller states, and $f$, an appropriate nonlinear function.

This closed-loop system has a manifold of exponentially stable equilibrium parametrized by the input signal $\overline{\mathbf{x}}(t)$, since it verifies the following properties:

(P1) the nonlinear function $f$ is twice continuously differentiable along a trajectory included in the wrench feasible workspace of the robot; 
(P2) there is a continuously differentiable equilibrium state $\overline{\mathbf{x}}_{c l}(\overline{\mathbf{x}}(t))=\left[\mathbf{0}^{T} \overline{\mathbf{x}}(t)^{T} \mathbf{0}^{T}\right]^{T}$ such that for each constant input value of $\overline{\mathbf{x}}, f\left(\overline{\mathbf{x}}_{c l}, \overline{\mathbf{x}}\right)=0$. This property can be verified by substituting $\overline{\mathbf{x}}_{c l}$ for $\mathbf{x}_{c l}$ in system of Eq. (27)-(28) and keep in mind that $\overline{\mathbf{l}}_{2}$ verifies $\mathbf{G}(\overline{\mathbf{x}})-$ $\tilde{\mathbf{J}}^{T} \mathbf{K}\left(\overline{\mathbf{l}}_{2}\right)\left[\overline{\mathbf{l}}_{2}-\mathbf{l}_{1}(\overline{\mathbf{x}})\right]=\mathbf{0}$ as a solution of the IKS model.

(P3) for each constant input value $\overline{\mathbf{x}}$, the linearized closed loop of the system (Eq. (27)-(28)) around the equilibrium $\left(\overline{\mathbf{x}}_{c l}(\overline{\mathbf{x}}), \overline{\mathbf{x}}\right)$ has eigenvalues with a negative real part. Actually, the linearization yields the system:

$$
\left\{\begin{array}{l}
\mathbf{M}(\overline{\mathbf{x}}) \Delta \ddot{\mathbf{x}}+\mathbf{K}_{\mathbf{x}}\left(\overline{\mathbf{x}}, \overline{\mathbf{l}}_{2}\right) \Delta \mathbf{x}=\mathbf{T}\left(\overline{\mathbf{x}}, \overline{\mathbf{l}}_{2}\right) \Delta \mathbf{l}_{2} \\
\Delta \mathbf{l}_{2}=\left.\frac{\partial \overline{\mathbf{l}}_{2}}{\partial \overline{\mathbf{x}}}\right|_{\left(\overline{\mathbf{x}}_{c l}, \overline{\mathbf{x}}\right)} \Delta \overline{\mathbf{x}}+\mathbf{T}^{+}\left(\mathbf{E}^{T}\right)^{-1} \mathbf{H}_{c}\left(\overline{\mathbf{x}}, \overline{\mathbf{l}}_{2}\right) \mathbf{x}_{c} \\
\dot{\mathbf{x}}_{c}=\mathbf{F}_{c}\left(\overline{\mathbf{x}}, \overline{\mathbf{l}}_{2}\right) \mathbf{x}_{c}+\mathbf{G}_{c} \mathbf{E}^{-1}\left(\overline{\mathbf{x}}, \overline{\mathbf{l}}_{2}\right) \Delta \dot{\mathbf{x}}
\end{array}\right.
$$

which has the same state matrix and thus eigenvalues than the linearized system of Eq. (25)-(26) around a constant equilibrium. Compared to Eq. (25)-(26), the system exhibits an additional input term in $\Delta \overline{\mathbf{x}}$. This term acts like an exogenous input disturbance (called "hidden coupling terms" in [33]). It is due to the dependence of the plant (CDPR) input signal on the time-varying signal $\overline{\mathbf{l}}_{2}(\overline{\mathbf{x}}(t))$ in order to track the trajectory.

The theorem [33], detailed in [34], thus states: for a given $\rho>0$, there are constants $\delta_{1}>0$ and $\delta_{2}>0$ such that if the input signal satisfies for $t>0,\|\dot{\overline{\mathbf{x}}}(t)\|<\delta_{2}$ and if

$$
\left\|\mathbf{x}_{c l}(0)-\overline{\mathbf{x}}_{c l}(0)\right\|=\left\|\left[\begin{array}{c}
\dot{\mathbf{x}}(0) \\
\mathbf{x}(0) \\
\mathbf{x}_{c}(0)
\end{array}\right]-\left[\begin{array}{c}
\mathbf{0} \\
\overline{\mathbf{x}}(0) \\
\mathbf{0}
\end{array}\right]\right\|<\delta_{1}
$$

then the solution of the system (Eq. (29)) verifies for $t>t_{0}$

$$
\left\|\mathbf{x}_{c l}(t)-\overline{\mathbf{x}}_{c l}(t)\right\|=\left\|\left[\begin{array}{c}
\dot{\mathbf{x}}(t) \\
\mathbf{x}(t) \\
\mathbf{x}_{c}(t)
\end{array}\right]-\left[\begin{array}{c}
\mathbf{0} \\
\overline{\mathbf{x}}(t) \\
\mathbf{0}
\end{array}\right]\right\|<\rho
$$

This result points out that, if the nonlinear closed loop system - composed of the CDPR (Eq. (27)) and gain scheduled controller (Eq. (28)) - is close enough to the equilibrium at time $t=0$ and if the input signal $\overline{\mathbf{x}}(t)$ does not vary too fast, then the system will remain in the neighborhood of the equilibrium trajectory defined by $\overline{\mathbf{x}}_{c l}(\overline{\mathbf{x}}(t))$. This neighborhood is defined by the open ball of radius $\rho$ and center $\overline{\mathbf{x}}_{c l}(\overline{\mathbf{x}}(t))$ as shown in Fig. 7. Extensive simulations can be carried out to estimate the numerical values of the bounds on the transient disturbance and trajectory velocity $\dot{\overline{\mathbf{x}}}$ in Fig. 5 that guarantee the stability property.

\section{Experiments}

\subsection{Experimental setup: CoMiRo}

A suspended 8-cables and 6-Degrees of Freedom (DoF) CDPR called CoMiRo has been built (Fig. 8). The geometry

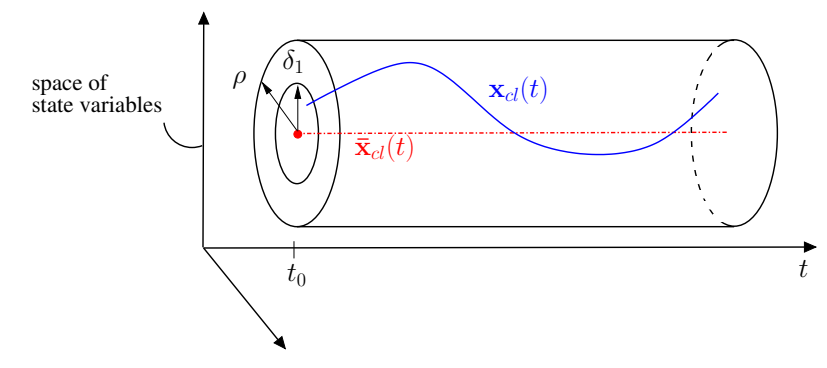

Fig. 7. Stability of the gain-scheduling modal damping along a trajectory: if $\overline{\mathbf{x}}(t)$ varies slowly, the closed loop system state $\mathbf{x}_{c l}(t)$ remains in the neighborhood of the equilibrium trajectory $\overline{\mathbf{x}}_{c l}(\overline{\mathbf{x}}(t))$

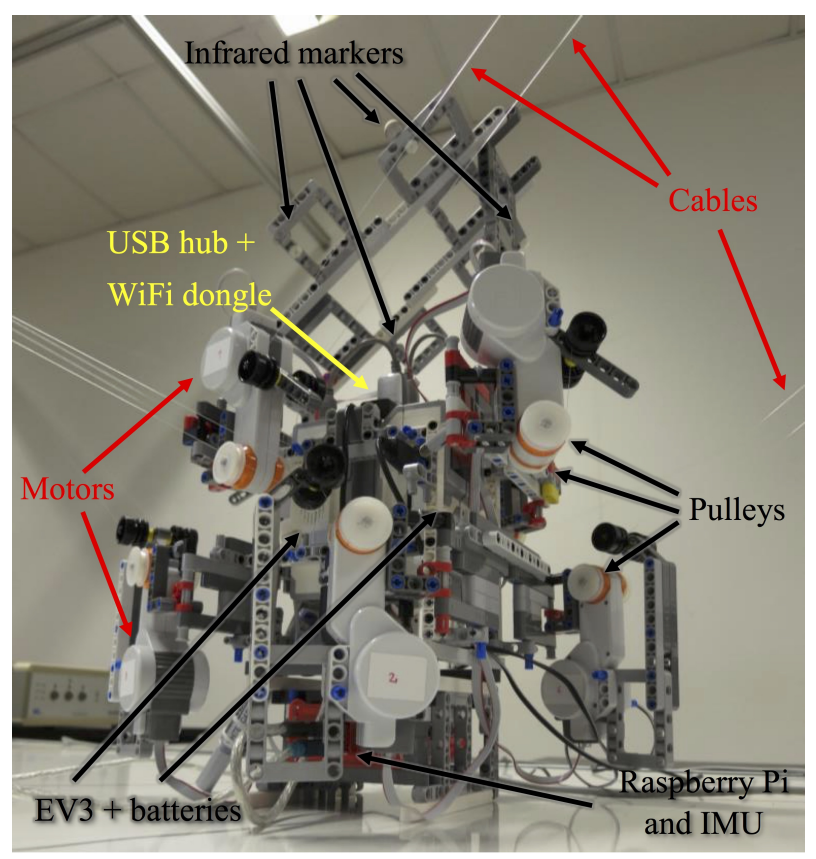

Fig. 8. CoMiRo robot

of the CoMiRo is similar to the geometry of the CoGiRo, a heavy payload CDPR developed in Montpellier (France) at the LIRMM by [3]. The CoMiRo cables anchor points on the room walls form a cuboid of 6.94 per 3.78 per 3.00 meters, along $\vec{x}, \vec{y}$ and $\vec{z}$ directions (see Fig. 9). The pulley output points at the end effector form another cuboid of 0.155 per 0.185 per 0.07 meters, along $\vec{x}, \vec{y}$ and $\vec{z}$ directions. The end effector weights $2 \mathrm{~kg}$.

It is built with Lego Mindstorms parts except for the cables, pulleys and some electronic components. Lego Mindstorms are an interesting solution to experiment with lightweight CDPRs for the following reasons:

- a rapid prototyping due to fast assembly and easily reconfigurable structure,

- a low cost, easily duplicable solution, with lightweight and compact components made of high-quality plastics embedding functions like motor drivers, sensors conditioners, power and logic,

- a worst case benchmark for control algorithms: due to the gearboxes and bearing sleeves made of plastic, high

Cuvillon, L., et al. 


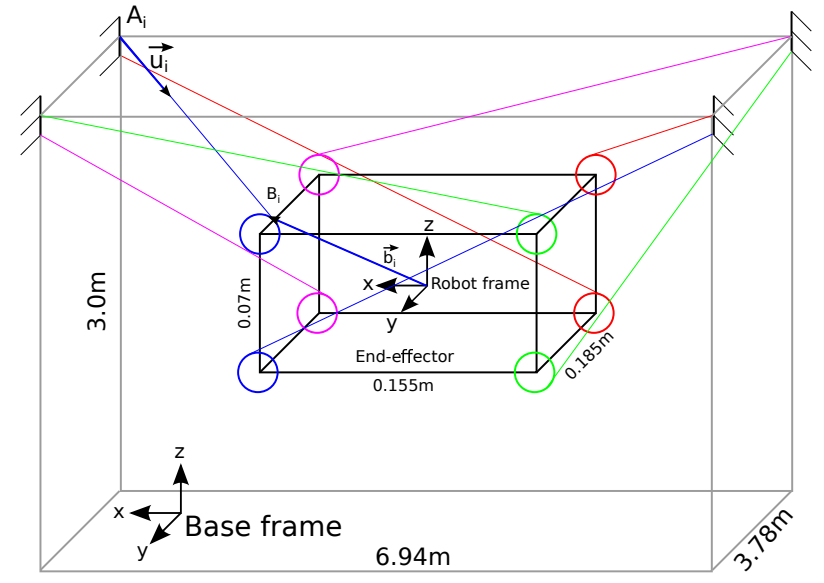

Fig. 9. CoMiRo notations, dimensions and frame definitions

nonlinear effects act on the Lego motors, such as quantization errors, backlash, and dry friction.

All the motors, sensors, computing power and batteries are embedded in the robot end effector including:

- an embedded computer providing a WiFi hotspot (Raspberry Pi model B),

- a 6-DoF Inertial Measurement Unit (Drotek IMU 9DOF - MPU9150 Invensense PCB),

- 2 Lego EV3 bricks,

- 8 Lego Mindstorms NXT actuators, each connected to a pair of 3D-printed pulleys.

Two cables (Shimano Blue Wing ${ }^{\mathrm{TM}}, 3.2 \mathrm{~kg}, 0.2 \mathrm{~mm}$, $\left.k_{s}=140.18 \mathrm{~N}\right)$ per actuator, instead of one, make the constraints on the pulley shaft more even, minimizing the frictions on the plastic sleeve bearing of the actuators. Each pair of cables is modeled as a single cable of specific stiffness $k_{s}=280.36 \mathrm{~N}$.

The robot controller, like the structure, is designed with a rapid prototyping approach for highly modular robotics applications. RPIt ${ }^{1}$ is a Simulink toolbox, developed in our laboratory, that uses the code generation feature of Simulink Coder $^{\mathrm{TM}}$ on a Raspberry Pi target. This toolbox provides all Simulink blocks needed for the experiment: multiple EV3 blocks, various IMU blocks, ... A custom firmware has been developed for the EV3 brick called EV3.14 ${ }^{2}$. It improves encoder resolution from 1 to $0.5^{\circ}$ and reduces USB communication delays between the brick and the Raspberry Pi from $5 \mathrm{~ms}$ to $1 \mathrm{~ms}$. Simulink external mode is used during the experiments to send motion commands, modify block parameters on the fly and monitor signals with scopes. A sampling interval of $7 \mathrm{~ms}$ is used in all experiments.

Each motor of the CoMiRo Lego is controlled by an independent position loop using encoder measurement feedback. Since this robot has 8 cables, the cable tension management algorithm should deal with a two-dimensional null space. Tensions can be obtained with the optimization method proposed in [31].

\footnotetext{
${ }^{1}$ http://icube-avr.unistra.fr/en/index.php/RPIt

${ }^{2}$ http://icube-avr.unistra.fr/en/index.php/EV3.14 Cuvillon, L., et al.
}

Furthermore, a NDI Polaris localizer was used to learn the current Cartesian pose $\overline{\mathbf{x}}(t)$ associated with the current cable free length $\overline{\mathbf{l}}_{2}(t)$. Indeed, the pose of the CDPR end effector cannot be estimated uniquely using only the sensors embedded into the end effector (motor encoders, IMU). However, after this learning phase, the proposed damping control law needs only the IMU feedback and does not require the localizer feedback anymore.

The proposed control law in Fig. 4 is implemented on the CoMiRo. Note that the translational velocity is obtained by integrating linear accelerations from IMU over time with a high-pass filter in order to avoid drifts. This velocity screw yields finally an estimate of $\dot{\mathbf{x}}$ using the definition of Eq. (3): $\dot{\mathbf{x}}=\mathbf{S}^{\prime-1}\left[\dot{\mathbf{p}}^{T} \omega^{T}\right]^{T}$.

\subsection{Modal Control Law Validation}

In this section, all experiments are done around a unique static equilibrium point $\overline{\mathbf{x}}_{1}$. It is located on the vertical symmetry axis of the robot workspace at a height $z=0.95 \mathrm{~m}$. Angles are set to zero, the base frame and robot frame are aligned. Euler angles $x y z$ are used to represent the rotational part of $\mathbf{x}$. At this pose, the matrix $\mathbf{K}_{\mathbf{x}}$ has the following value:

$$
\mathbf{K}_{\mathbf{x}}=\left(\begin{array}{cccccc}
316.3 & 0 & 0 & -0.24 & 11.18 & 0 \\
0 & 101.2 & 0 & -4.10 & 0.24 & 0 \\
0 & 0 & 110.0 & 0 & 0 & 0 \\
-0.24 & -4.10 & 0 & 1.81 & -0.32 & 0 \\
11.18 & 0.24 & 0 & -0.32 & 2.09 & 0 \\
0 & 0 & 0 & 0 & 0 & 5.57
\end{array}\right)
$$

To compute $\mathbf{K}_{\mathbf{x}}$, the tension at equilibrium $\bar{\tau}$ is needed (Eq. (9)). This tension is directly related to the cable elongation obtained from the pose $\overline{\mathbf{x}}_{1}$ and the cable free length $\overline{\mathbf{l}}_{2}$ measured by the winch encoders (Eq. (5)).

With the inertia tensor $\mathbf{I}_{\mathbf{r}}$ expressed in the robot frame, the mass matrix $\mathbf{M}$ of the end effector takes the following value:

$\mathbf{M}=\left(\begin{array}{cc}2.0 \mathbb{I}_{3 \times 3} & \mathbf{0} \\ \mathbf{0} & \mathbf{I}_{\mathbf{r}}\end{array}\right) \quad$ with $\mathbf{I}_{\mathbf{r}}=\left(\begin{array}{ccc}0.0138 & 0 & 0 \\ 0 & 0.121 & 0 \\ 0 & 0 & 0.0049\end{array}\right)$

Knowing $\mathbf{K}_{\mathbf{x}}$ and $\mathbf{M}$, modal matrices $\mathbf{E}$ and $\boldsymbol{\Lambda}$ are computed using the method described by Eq. (13).

$$
\mathbf{E}=\left(\begin{array}{cccccc}
0 & 0.45 & -0.25 & -0.48 & 0 & 0.03 \\
0 & 0.02 & 0.18 & -0.11 & 0 & -0.68 \\
0 & 0 & 0 & 0 & -0.71 & 0 \\
0 & -1.57 & -7.66 & 2.36 & 0 & -2.43 \\
0 & 6.77 & 0.66 & 6.02 & 0 & -0.58 \\
14.35 & 0 & 0 & 0 & 0 & 0
\end{array}\right)
$$

The modal frequencies $\mathbf{F}_{\mathbf{m}}$ of the robot are the square root of the eigenvalues $\Lambda_{i}$ in $\Lambda$. Furthermore, the mode corresponding to the $i^{\text {th }}$ frequency in $\mathbf{F}_{\mathbf{m}}$ (in $\left.\mathrm{rad} \mathrm{s}^{-1}\right)$ has the direction of 


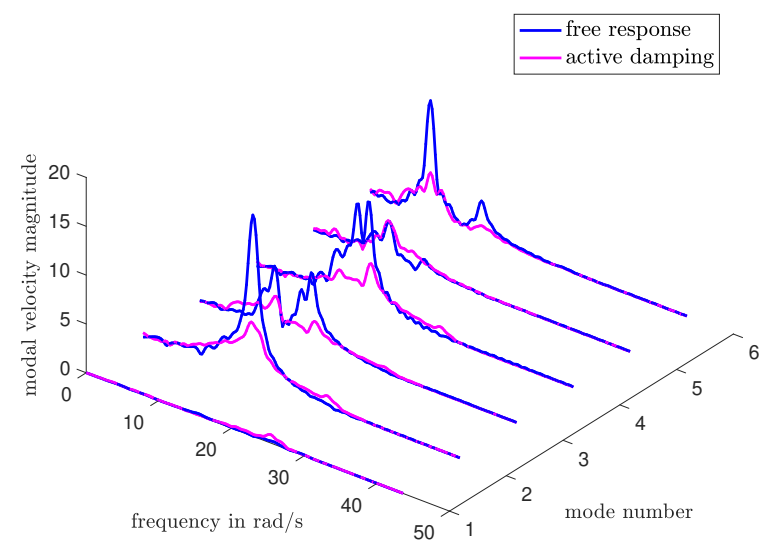

Fig. 10. Frequency-domain response of the CDPR to a pulse disturbance at pose $\overline{\mathbf{x}}_{1}$

the $i^{\text {th }}$ column in $\mathbf{E}$.

$$
\mathbf{F}_{\mathbf{m}}=\left(\begin{array}{c}
33.87 \\
15.55 \\
11.82 \\
9.41 \\
7.40 \\
6.58
\end{array}\right)
$$

Experimental results of the modal vibration damping control loop in the frequency domain are shown in Fig. 10. An impulse disturbance is added to the signal $\mathbf{u}_{\mathbf{m}}$ (see Fig. 4) to mainly excite vibration mode 2 . With the proposed modal control, the residual vibration magnitude on all modes is significantly reduced compared to the free response.

\subsection{Gain Scheduling \\ 5.3.1 Motivations}

To validate the need for a gain scheduling approach like the one proposed in Eq. (28), the controller parameters calculated at the pose $\overline{\mathbf{x}}_{1}$ defined in the previous section are frozen and performance of the disturbance damping control law is assessed after moving the robot at a significantly different pose without updating the controller. This assessment is referred as "Active" or "Active damping" in what follows. Then, the controller is updated according to this new position and a new performance assessment is made. This is referred as "GS" or "Gain Scheduling" in what follows.

Thanks to its simple form presented in section 3.4, the control algorithm can be easily and quickly updated online accordingly to this model within the sampling time of $7 \mathrm{~ms}$ :

- the matrix $\mathbf{T}^{+}\left(\mathbf{E}^{T}\right)^{-1}\left(\overline{\mathbf{x}}(t), \overline{\mathbf{l}}_{2}(t)\right)$ is updated in the control loop of Fig. 4 to convert modal control signals into actuators signals;

- and each SISO controller is updated as detailed in section 3.4 according to the current value of the modal frequencies matrix $\Lambda$.
Table 1. RMS errors on modal velocities (without unit) at pose $\overline{\mathbf{x}}_{2}$ for a velocity impulse disturbances on mode 1 and 3

\begin{tabular}{|c||c|c|c|c|c|c|c|}
\hline Modes & 1 & 2 & 3 & 4 & 5 & 6 & $\sum$ modes \\
\hline Free & 9.31 & 1.47 & 6.10 & 0.98 & 0.40 & 2.99 & 21.25 \\
\hline Active & 4.66 & 2.40 & 3.79 & 3.25 & 0.59 & 2.86 & 17.55 \\
\hline GS & 4.25 & 0.83 & 2.57 & 1.04 & 0.24 & 1.70 & 10.63 \\
\hline
\end{tabular}

On the CoMiRo robot, modal frequencies vary from $4.8 \mathrm{rad} \mathrm{s}^{-1}$ to $41.9 \mathrm{rad} \mathrm{s}^{-1}$ inside a Static Feasible Workspace defined by a $4.5 \times 1.5 \times 2 \mathrm{~m}^{3}$ cuboid centered in $\overline{\mathbf{x}}_{1}$ where end-effector Euler angles vary from $-15^{\circ}$ to $+15^{\circ}$. The modal frequencies are evaluated on discrete poses within the workspace with translational quantization steps of $0.1 \mathrm{~m}$ and angular steps of $5^{\circ}$. To select an admissible equilibrium tension at each point, the tension distribution algorithm proposed in [31] with minimization of the $\mathcal{L}_{2}$ tension norm is used. The lower admissible cable tension is $0.1 \mathrm{~N}$ and the upper one is $17.5 \mathrm{~N}$.

\subsubsection{Results}

Table 1 and Fig. 11 show the added value of the gainscheduling approach for vibration damping at a static equilibrium pose $\overline{\mathbf{x}}_{2}$, far from the pose $\overline{\mathbf{x}}_{1}$ where the active damping has been computed. The pose $\overline{\mathbf{x}}_{2}$ chosen for this experiment is translated by $\overline{\mathbf{x}}_{2}-\overline{\mathbf{x}}_{1}=[-2,0,1]^{T}$ meters from the workspace center pose $\overline{\mathbf{x}}_{1}$ defined in the previous section. Measured velocity data are projected into modal space with the modal matrix $\mathbf{E}$ computed at the current pose 2 . The figures $11 \mathrm{~d}$ and $11 \mathrm{e}$ show that performance degrades when the controller is not tuned ("active damping") to the mode frequency of the current pose: instead of being damped, vibrations on modes 4 and 5 are amplified. This degradation is also visible in the frequency domain as shown in Fig. 12. Thus, gain-scheduling control is required to guarantee optimal damping over the whole workspace.

The figures 14 and 15 compare the performance of the proposed control strategy without and with gain-scheduling when the suspended robot is moving at a constant velocity along $x$ axis (Fig. 13). The maximum translational velocity is $10 \mathrm{~cm} \mathrm{~s}^{-1}$ due to limitations of the Lego actuators. To highlight the vibration rejection, a modal velocity impulse disturbance is applied simultaneously on the 6 modes. The result is a clear reduction of the vibrations (Fig. 15 compared to Fig. 14) for all modes when a gain scheduling control law is activated during the whole trajectory. This vibration damping is also visible in the frequency domain as shown in Fig. 16. The variations $\Delta \theta$ of the winch angular positions yielding cable tension variations that damp the CDPR vibrations with the GS controller are given at the bottom of Fig. 15. This control signal issued by the modal controller is used as reference for the inner loop that controls the unstretched cable length $\Delta \mathbf{l}_{2}=r \Delta \boldsymbol{\theta}$ with $r$ the winch radius.

The video of this experiment can also be viewed online at https://youtu.be/tbovdrJk3Nk. At time 00:02:50, two superimposed videos are displayed: the same 


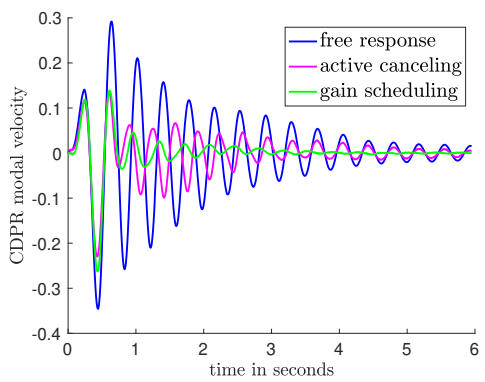

(a) Mode 1

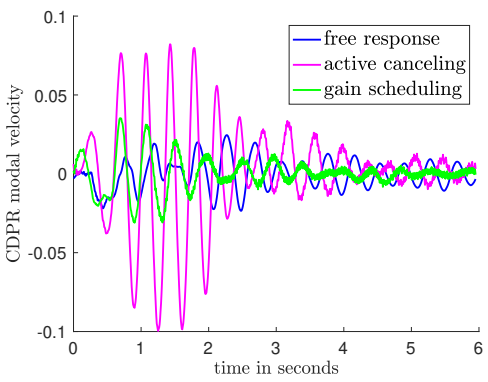

(d) Mode 4

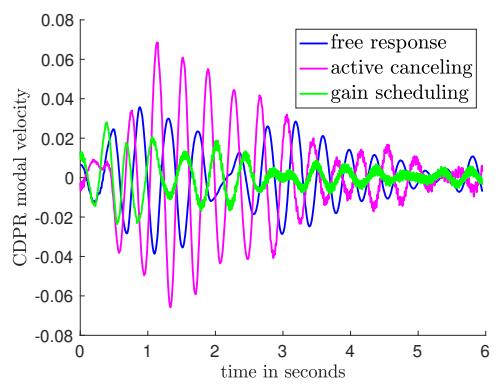

(b) Mode 2

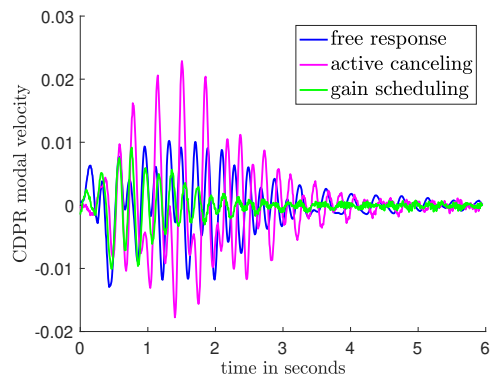

(e) Mode 5

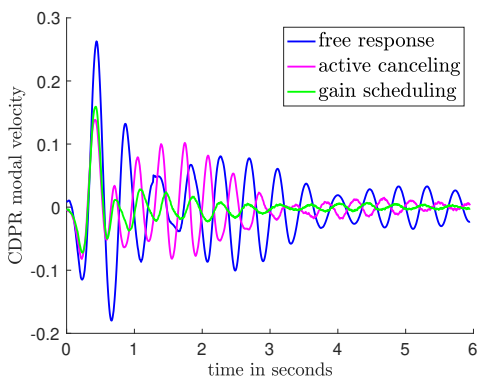

(c) Mode 3

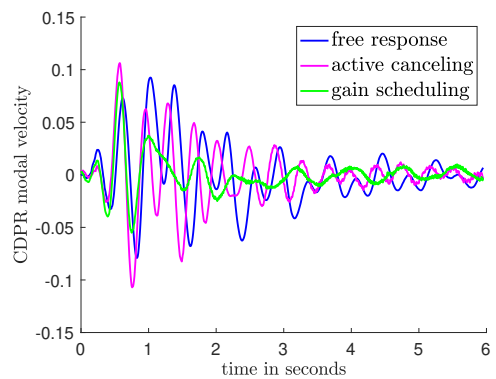

(f) Mode 6

Fig. 11. Modal damping assessment with a velocity impulse disturbances on mode 1 and 3 at pose $\overline{\mathbf{x}}_{2}$

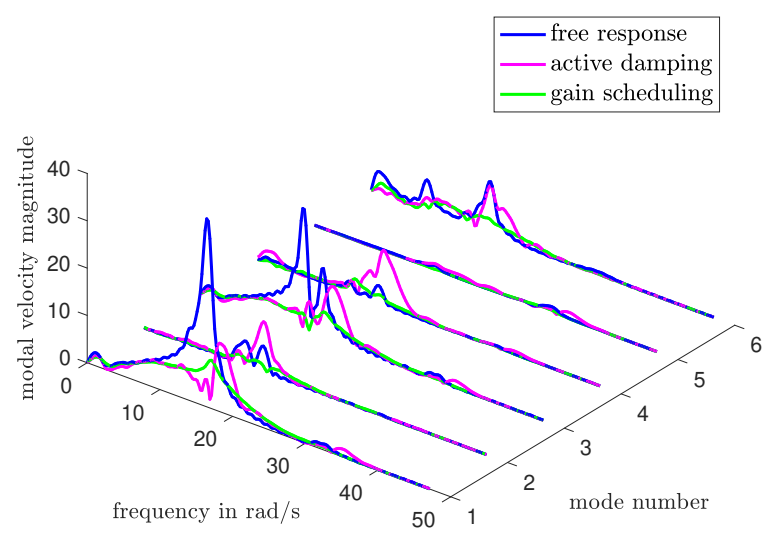

Fig. 12. Frequency-domain response of the CDRP to a pulse disturbance on mode 1 and 3 at pose $\overline{\mathbf{x}}_{2}$

motion with and without active damping.

\section{Conclusions}

Using modal analysis, a linear decoupled model of a CDPR with elastic cables is described. Projecting the robot linearized dynamics into the modal space yields 6 decoupled linear second order transfer functions which can be easily controlled with standard SISO techniques. This is the main benefit of the proposed approach. A control strategy, that cancels the vibrations of the end effector caused by disturbances is proposed. Furthermore, thanks to the decoupled nature of the model, the modal controller can be easily up-

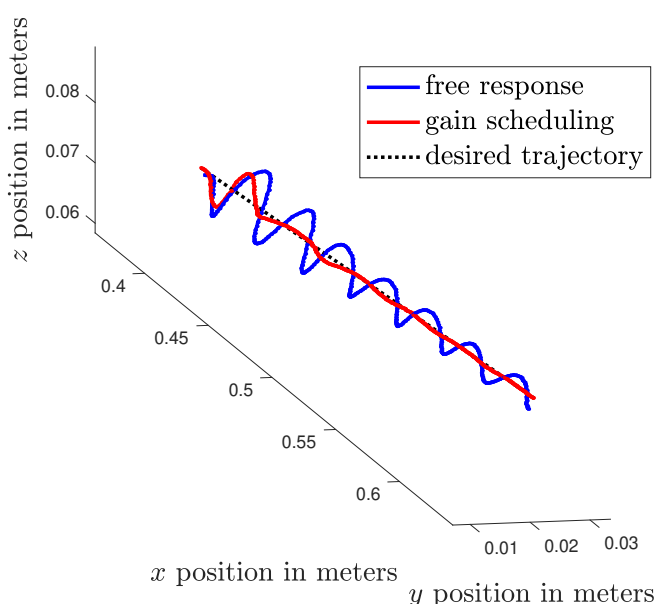

Fig. 13. Cartesian trajectory of CDPR end effector during a translation motion without modal damping (free) and with gain scheduling modal damping. A impulse modal disturbance is applied on all the vibration mode at one point of the trajectory to highlight the vibration rejection.

dated with a trivial, computational efficient algorithm. Since natural modes and frequencies are related to the end effector pose, a gain-scheduling approach is introduced to improve the efficiency of the vibration damping algorithm over the whole workspace of the CDPR. Finally, stability analysis shows that the control law remains stable along a slowly varying trajectory. The proposed approach is validated experimentally on a small suspended CDPR built with Lego Mindstorms, which shares the same architecture as its big

Cuvillon, L., et al. 

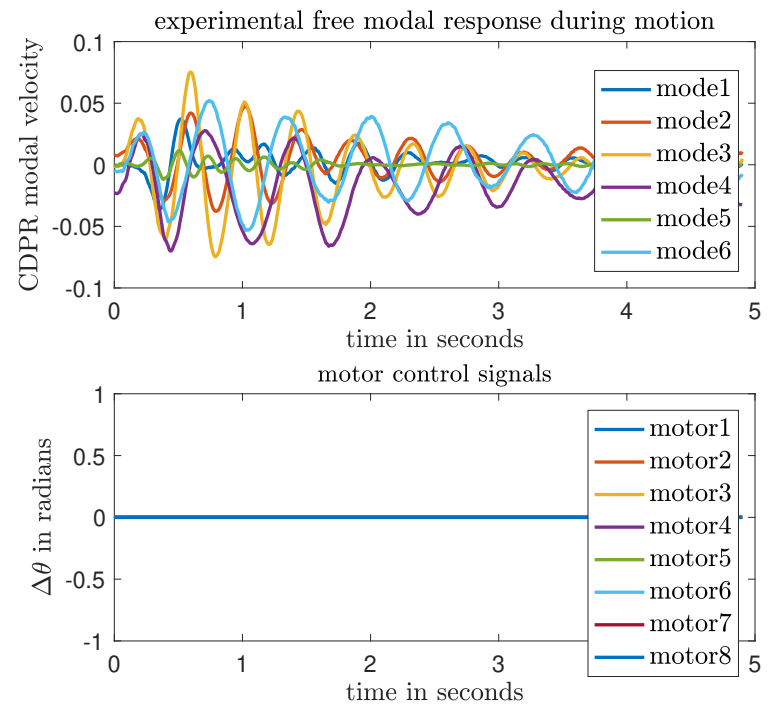

Fig. 14. Vibration in the modal space during motion in the absence of active damping (on top) and associate position motor control signal $\Delta \boldsymbol{\theta}$ (on bottom) issued by the modal controller (inactive here)

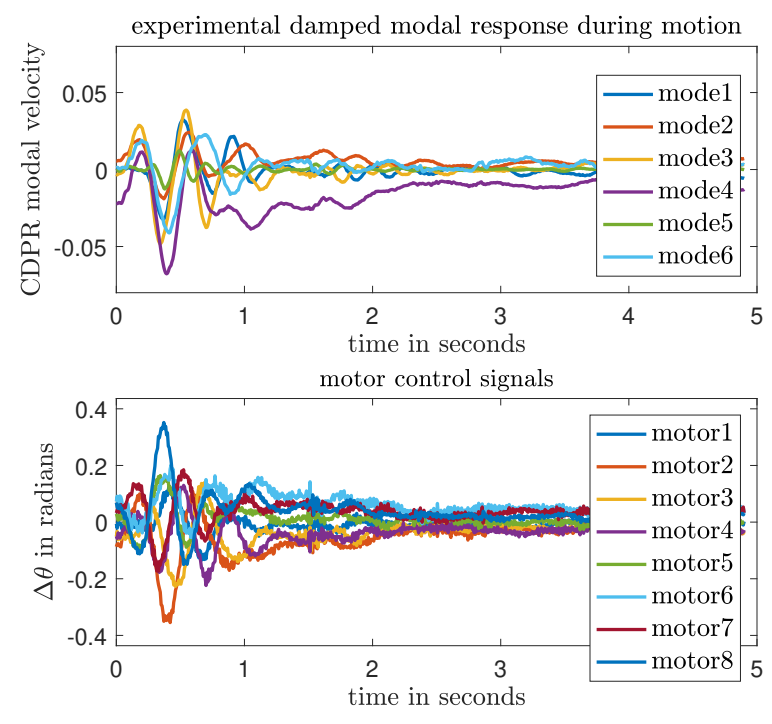

Fig. 15. Vibration in the modal space during motion with gainscheduling modal damping (on top) and associate position motor control signal $\Delta \boldsymbol{\theta}$ issued by the modal controller (on the bottom)

brother, the CoGiRo robot (LIRMM, Montpellier, France).

\section{References}

[1] Thompson, R., and Blackstone, M., 2005. Threedimensional moving camera assembly with an informational cover housing, Mar. 29. US Patent 6,873,355.

[2] Merlet, J.-p., and Daney, D., 2010. "A portable, modular parallel wire crane for rescue operations". In 2010 IEEE International Conference on Robotics and $\mathrm{Au}-$ tomation, Anchorage, AK, May 3-7, pp. 2834-2839.

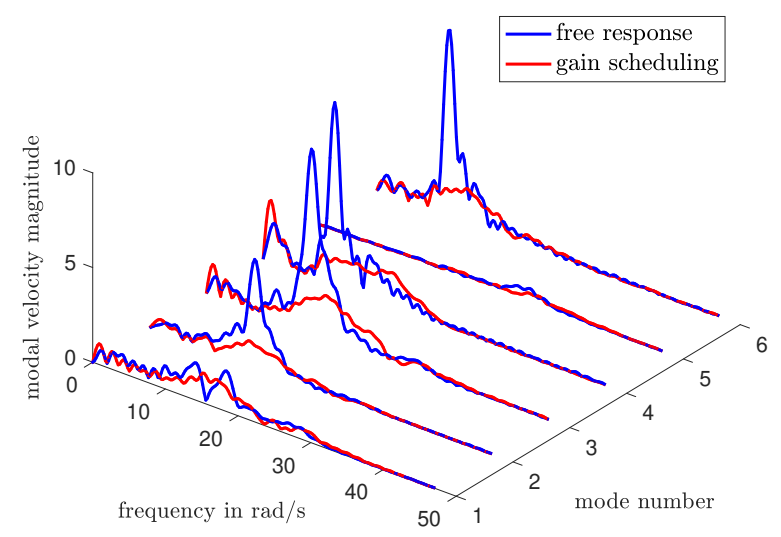

Fig. 16. Frequency-domain response of the CDPR to a pulse disturbance on all modes applied at one point of the trajectory

[3] Gouttefarde, M., Collard, J.-F., Riehl, N., and Baradat, C., 2015. "Geometry Selection of a Redundantly Actuated Cable-Suspended Parallel Robot". IEEE Transactions on Robotics, 31(2), Apr., pp. 501-510.

[4] Gouttefarde, M., Collard, J.-F., Riehl, N., and Baradat, C., 2012. "Simplified static analysis of large-dimension parallel cable-driven robots". In 2012 IEEE International Conference on Robotics and Automation, Saint Paul, MN, May 14-18, pp. 2299-2305.

[5] Kawamura, S., Choe, W., Tanaka, S., and Pandian, S., 1995. "Development of an ultrahigh speed robot FALCON using wire drive system". In Proceedings of 1995 IEEE International Conference on Robotics and $\mathrm{Au}-$ tomation, Nagoya, Japan, May 21-27, Vol. 1, pp. 215220.

[6] Weber, X., Cuvillon, L., and Gangloff, J., 2014. "Active vibration canceling of a cable-driven parallel robot using reaction wheels". In 2014 IEEE/RSJ International Conference on Intelligent Robots and Systems, Chicago, IL, September 14-18, pp. 1724-1729.

[7] de Rijk, R., Rushton, M., and Khajepour, A., 2018. "Out-of-plane vibration control of a planar cable-driven parallel robot". IEEE/ASME Transactions on Mechatronics, 23(4), Aug, pp. 1684-1692.

[8] Sellet, H., Khayour, I., Cuvillon, L., Durand, S., and Gangloff, J., 2019. "Active damping of parallel robots driven by flexible cables using cold-gas thrusters". In 2019 IEEE International Conference on Robotics and Automation, Montreal, QC, May 20-24, pp. 530-536.

[9] Yanai, N., Yamamoto, M., and Mohri, A., 2002. "Antisway control for wire-suspended mechanism based on dynamics compensation". In Proceedings 2002 IEEE International Conference on Robotics and Automation, Washington, DC, May 11-15, Vol. 4, pp. 4287-4292.

[10] Caverly, R. J., and Forbes, J. R., 2014. "Dynamic modeling and noncollocated control of a flexible planar cable-driven manipulator". IEEE Transactions on Robotics, 30(6), Dec, pp. 1386-1397.

[11] Khosravi, M., and Taghirad, H., 2014. "Dynamic Mod- 
eling and Control of Parallel Robots With Elastic Cables: Singular Perturbation Approach". IEEE Transactions on Robotics, 30(3), June, pp. 694-704.

[12] Khosravi, M. A., and Taghirad, H. D., 2016. "Stability analysis and robust pid control of cable-driven robots considering elasticity in cables". Amirkabir International Journal of Electrical and Electronics Engineering, 48(2), pp. 113-125.

[13] Begey, J., Cuvillon, L., Lesellier, M., Gouttefarde, M., and Gangloff, J., 2019. "Dynamic control of parallel robots driven by flexible cables and actuated by position-controlled winches". IEEE Transactions on Robotics, 35(1), Feb, pp. 286-293.

[14] Meunier, G., Boulet, B., and Nahon, M., 2009. “Control of an overactuated cable-driven parallel mechanism for a radio telescope application". IEEE Transactions on Control Systems Technology, 17(5), Sept, pp. 10431054.

[15] Jamshidifar, H., Khosravani, S., Fidan, B., and Khajepour, A., 2018. "Vibration decoupled modeling and robust control of redundant cable-driven parallel robots". IEEE/ASME Transactions on Mechatronics, 23(2), April, pp. 690-701.

[16] Baklouti, S., Courteille, E., Lemoine, P., and Caro, S., 2019. "Vibration reduction of cable-driven parallel robots through elasto-dynamic model-based control". Mechanism and Machine Theory, 139, pp. 329 - 345.

[17] Ghorbel, F., Hung, J. Y., and Spong, M. W., 1989. "Adaptive control of flexible-joint manipulators". IEEE Control Systems Magazine, 9(7), Dec, pp. 9-13.

[18] Khalil, H., 2001. Nonlinear Systems, Second Edition. Prentice-Hall.

[19] Behzadipour, S., and Khajepour, A., 2005. "Stiffness of Cable-based Parallel Manipulators With Application to Stability Analysis". Journal of Mechanical Design, 128(1), Apr., pp. 303-310.

[20] Diao, X., and Ma, O., 2009. "Vibration analysis of cable-driven parallel manipulators". Multibody System Dynamics, 21(4), May, pp. 347-360.

[21] Yuan, H., Courteille, E., and Deblaise, D., 2015. "Static and dynamic stiffness analyses of cable-driven parallel robots with non-negligible cable mass and elasticity". Mechanism and Machine Theory, 85, pp. 64-81.

[22] Gould, L., and Murray-Lasso, M., 1966. "On the modal control of distributed systems with distributed feedback". IEEE Transactions on Automatic Control, 11(4), Oct., pp. 729-737.

[23] Preumont, A., 2011. "Vibration Control of Active Structures". In Vibration Control of Active Structures, G. M. L. Gladwell, ed., Vol. 179 of Solid Mechanics and Its Applications. Springer Netherlands, Dordrecht.

[24] Singh, S. P., Singh Pruthi, H., and Agarwal, V. P., 2003. "Efficient modal control strategies for active control of vibrations". Journal of Sound and Vibration, 262(3), May, pp. 563-575.

[25] Wang, X., Mills, J., and Guo, S., 2009. "Experimental Identification and Active Control of Configuration Dependent Linkage Vibration in a Planar Parallel
Robot”. IEEE Transactions on Control Systems Technology, 17(4), July, pp. 960-969.

[26] Kozak, K., Ebert-Uphoff, I., and Singhose, W., 2004. "Locally Linearized Dynamic Analysis of Parallel Manipulators and Application of Input Shaping to Reduce Vibrations". Journal of Mechanical Design, 126(1), Mar., pp. 156-168.

[27] Weber, X., Cuvillon, L., and Gangloff, J., 2015. “Active vibration canceling of a cable-driven parallel robot in modal space". In 2015 IEEE International Conference on Robotics and Automation, Seattle, WA, May 26-30, pp. 1599-1604.

[28] Riehl, N., Gouttefarde, M., Baradat, C., and Pierrot, F., 2010. "On the determination of cable characteristics for large dimension cable-driven parallel mechanisms". In 2010 IEEE International Conference on Robotics and Automation, Anchorage, AK, May 3-7, pp. $4709-4714$.

[29] Alp, A., and Agrawal, S., 2002. "Cable suspended robots: feedback controllers with positive inputs". In Proceedings of the 2002 American Control Conference, Anchorage, AK, May 8-10, Vol. 1, pp. 815-820.

[30] Oh, S.-R., and Agrawal, S., 2005. "Cable suspended planar robots with redundant cables: controllers with positive tensions". IEEE Transactions on Robotics, 21(3), June, pp. 457-465.

[31] Gouttefarde, M., Lamaury, J., Reichert, C., and Bruckmann, T., 2015. "A versatile tension distribution algorithm for $n$-dof parallel robots driven by $n+2$ cables". IEEE Transactions on Robotics, 31(6), Dec, pp. 14441457.

[32] Azadi, M., Behzadipour, S., and Faulkner, G., 2009. "Antagonistic variable stiffness elements". Mechanism and Machine Theory, 44(9), Sept., pp. 1746-1758.

[33] Rugh, W. J., and Shamma, J. S., 2000. "Research on gain scheduling". Automatica, 36(10), Oct., pp. 14011425.

[34] Lawrence, D. A., and Rugh, W. J., 1990. “On a stability theorem for nonlinear systems with slowly varying inputs”. IEEE Transactions on Automatic Control, 35(7), Jul, pp. 860-864. 


\section{A Appendix: CDPR Linearization Derivation}

$$
\begin{aligned}
& \mathbf{M}(\overline{\mathbf{x}}) \Delta \ddot{\mathbf{x}}+\left[\mathbf{C}(\dot{\overline{\mathbf{x}}}, \overline{\mathbf{x}})+\left.\frac{\partial \mathbf{C}(\dot{\mathbf{x}}, \mathbf{x})}{\partial \dot{\mathbf{x}}}\right|_{\dot{\mathbf{x}}, \dot{\mathbf{x}}} \dot{\overline{\mathbf{x}}}\right] \Delta \dot{\mathbf{x}} \\
& -\left[\left.\tilde{\mathbf{J}}^{T}(\overline{\mathbf{x}}) \frac{\partial \mathbf{K}\left(\mathbf{l}_{2}\right)}{\partial \mathbf{l}_{2}}\right|_{\mathbf{I}_{2}}\left[\overline{\mathbf{I}}_{2}-\mathbf{l}_{1}(\overline{\mathbf{x}})\right]+\right. \\
& \left.\left.\tilde{\mathbf{J}}^{T}(\overline{\mathbf{x}}) \mathbf{K}\left(\overline{\mathbf{l}}_{2}\right) \frac{\partial\left[\mathbf{l}_{2}-\mathbf{l}_{1}(\mathbf{x})\right]}{\partial \mathbf{l}_{2}}\right|_{\overline{\mathbf{x}}, \overline{\mathbf{l}}_{2}}\right] \Delta \mathbf{l}_{2} \\
& +\left[\left.\frac{\partial \mathbf{M}(\mathbf{x})}{\partial \mathbf{x}}\right|_{\overline{\mathbf{x}}} \ddot{\overline{\mathbf{x}}}+\left.\frac{\partial \mathbf{C}(\dot{\mathbf{x}}, \mathbf{x})}{\partial \mathbf{x}}\right|_{\dot{\mathbf{x}}, \overline{\mathbf{x}}} \dot{\overline{\mathbf{x}}}-\right. \\
& \left.\frac{\partial \tilde{\mathbf{J}}^{T}(\mathbf{x})}{\partial \mathbf{x}}\right|_{\overline{\mathbf{x}}} \underbrace{\mathbf{K}\left(\overline{\mathbf{l}}_{2}\right)\left[\overline{\mathbf{l}}_{2}-\mathbf{l}_{1}(\overline{\mathbf{x}})\right]}_{-\bar{\tau}}- \\
& \tilde{\mathbf{J}}^{T}(\overline{\mathbf{x}}) \mathbf{K}\left(\overline{\mathbf{l}}_{2}\right) \underbrace{\left.\frac{\partial\left(-\mathbf{l}_{1}(\mathbf{x})\right)}{\partial \mathbf{x}}\right|_{\overline{\mathbf{x}}}}_{-\tilde{\mathbf{J}}(\overline{\mathbf{x}})}] \Delta \mathbf{x}-\Delta \boldsymbol{\psi}=\mathbf{0}
\end{aligned}
$$

And using:

$$
\begin{array}{r}
\left.\left.\frac{\partial \mathbf{K}\left(\mathbf{l}_{2}\right)}{\partial \mathbf{l}_{2}}\right|_{\mathbf{I}_{2}}\left[\overline{\mathbf{l}}_{2}-\mathbf{l}_{1}(\overline{\mathbf{x}})\right)\right]=\left.\frac{\partial \operatorname{diag}^{-1}\left(\mathbf{l}_{2}\right)}{\partial \mathbf{l}_{2}}\right|_{\overline{\mathbf{l}}_{2}} \operatorname{diag}(e s)\left[\overline{\mathbf{l}}_{2}-\mathbf{l}_{1}(\overline{\mathbf{x}})\right] \\
=-\left.\operatorname{diag}^{-1}\left(\overline{\mathbf{l}}_{2}\right) \frac{\partial \operatorname{diag}\left(\mathbf{l}_{2}\right)}{\partial \mathbf{l}_{2}}\right|_{\overline{\mathbf{l}}_{2}} \operatorname{diag}^{-1}\left(\overline{\mathbf{l}}_{2}\right) \operatorname{diag}(e s)\left[\overline{\mathbf{l}}_{2}-\mathbf{l}_{1}(\overline{\mathbf{x}})\right] \\
=-\left.\operatorname{diag}^{-1}\left(\overline{\mathbf{l}}_{2}\right) \frac{\partial \operatorname{diag}\left(\mathbf{l}_{2}\right)}{\partial \mathbf{l}_{2}}\right|_{\mathbf{l}_{2}} \underbrace{\mathbf{K}\left(\overline{\mathbf{l}}_{2}\right)\left[\overline{\mathbf{l}}_{2}-\mathbf{l}_{1}(\overline{\mathbf{x}})\right]}_{-\overline{\boldsymbol{\tau}}} \\
\left.\frac{\partial \mathbf{K}\left(\mathbf{l}_{2}\right)}{\partial \mathbf{l}_{2}}\right|_{\mathbf{l}_{2}}\left[\overline{\mathbf{l}}_{2}-\mathbf{l}_{1}(\overline{\mathbf{x}})\right]=\operatorname{diag}^{-1}\left(\overline{\mathbf{l}}_{2}\right) \operatorname{diag}(\overline{\boldsymbol{\tau}})
\end{array}
$$

(since for $u$ scalar, $\frac{\partial A^{-1}}{\partial u}=-A^{-1} \frac{\partial A}{\partial u} A^{-1}$ ) finally yields Eq. (7).

\section{List of Figures}

1 CDPR notations . . . . . . . . . . 3

2 Architecture of the vibration damping control loop . . . . . . . . . . . . 5

3 Vibration damping control loop scheme in modal space . . . . . . . . . . . . 5

4 Practical implementation of the modal vibration damping control on the CDPR . . . . . . 6

5 Block diagram of the CDPR nominal trajectory generation (left side) combined with the vibration damping control (right side) . . . . 6

6 Root locus design of a modal controller . . . 6

7 Stability of the gain-scheduling modal damping along a trajectory: if $\overline{\mathbf{x}}(t)$ varies slowly, the closed loop system state $\mathbf{x}_{c l}(t)$ remains in the neighborhood of the equilibrium trajectory $\overline{\mathbf{x}}_{c l}(\overline{\mathbf{x}}(t)) \ldots \ldots \ldots \ldots$

$8 \quad$ CoMiRo robot . . . . . . . . . . 8

9 CoMiRo notations, dimensions and frame definitions ............... 9
10 Frequency-domain response of the CDPR to a pulse disturbance at pose $\overline{\mathbf{x}}_{1} \ldots \ldots \ldots$

11 Modal damping assessment with a velocity impulse disturbances on mode 1 and 3 at pose $\overline{\mathbf{x}}_{2} \ldots \ldots \ldots \ldots \ldots \ldots$

12 Frequency-domain response of the CDRP to a pulse disturbance on mode 1 and 3 at pose $\overline{\mathbf{x}}_{2} \quad 11$

13 Cartesian trajectory of CDPR end effector during a translation motion without modal damping (free) and with gain scheduling modal damping. A impulse modal disturbance is applied on all the vibration mode at one point of the trajectory to highlight the vibration rejection. . . . . . . . . 11

14 Vibration in the modal space during motion in the absence of active damping (on top) and associate position motor control signal $\Delta \boldsymbol{\theta}$ (on bottom) issued by the modal controller (inactive here) . . . . . . . . . 12

15 Vibration in the modal space during motion with gain-scheduling modal damping (on top) and associate position motor control signal $\Delta \boldsymbol{\theta}$ issued by the modal controller (on the bottom) . . . . . . . . . . . 12

16 Frequency-domain response of the CDPR to a pulse disturbance on all modes applied at one point of the trajectory . . . . . . . .

\section{List of Tables}

1 RMS errors on modal velocities (without unit) at pose $\overline{\mathbf{x}}_{2}$ for a velocity impulse disturbances on mode 1 and $3 \ldots \ldots$

Cuvillon, L., et al. 